Draft VERSION JunE 8, 2018

Preprint typeset using $\mathrm{LATEX}_{\mathrm{E}}$ style emulateapj v. 08/13/06

\title{
NUMERICAL TESTS OF FAST RECONNECTION IN WEAKLY STOCHASTIC MAGNETIC FIELDS
}

\author{
Grzegorz KowAL \\ Department of Astronomy, University of Wisconsin, 475 North Charter Street, Madison, WI 53706, USA and \\ Astronomical Observatory, Jagiellonian University, Orla 171, 30-244 Kraków, POLAND \\ AND \\ A. LAZARIAN \\ Department of Astronomy, University of Wisconsin, 475 North Charter Street, Madison, WI 53706, USA \\ AND \\ E. T. VishniaC \\ Department of Physics and Astronomy, McMaster University, 1280 Main Street West, Hamilton, ON L8S 4M1, CANADA \\ AND \\ K. Otmianowska-Mazur \\ Astronomical Observatory, Jagiellonian University, Orla 171, 30-244 Kraków, POLAND \\ Draft version June 8, 2018
}

\begin{abstract}
We study the effects of turbulence on magnetic reconnection using three-dimensional direct numerical simulations. This is the first attempt to test a model of fast magnetic reconnection in the presence of weak turbulence proposed by Lazarian \& Vishniac (1999). This model predicts that weak turbulence, which is generically present in most of astrophysical systems, enhances the rate of reconnection by reducing the transverse scale for reconnection events and by allowing many independent flux reconnection events to occur simultaneously. As a result the reconnection speed becomes independent of Ohmic resistivity and is determined by the magnetic field wandering induced by turbulence. We test the dependence of the reconnection speed on turbulent power, the energy injection scale and resistivity. We study the reconnection model with the open and experiment with the outflow boundary conditions and discuss the advantages and drawbacks of various setups. To test our results, we also perform simulations of turbulence with the same outflow boundaries but without a large scale field reversal, thus without large scale reconnection. To quantify the reconnection speed we use both an intuitive definition, i.e. the speed of the reconnected flux inflow, as well as a more sophisticated definition based on a formally derived analytical expression. Our results confirm the predictions of the Lazarian \& Vishniac model. In particular, we find that the reconnection speed is proportional to the square root of the injected power, as predicted by the model. The dependence on the injection scale for some of our models is a bit weaker than expected, i.e. $l_{i n j}^{3 / 4}$, compared to the predicted linear dependence on the injection scale, which may require some refinement of the model or may be due to the effects like finite size of the excitation region, which are not a part of the model. The reconnection speed was found to depend on the expected rate of magnetic field wandering and not on the magnitude of the guide field. In our models, we see no dependence on the guide field when its strength is comparable to the reconnected component. More importantly, while in the absence of turbulence we successfully reproduce the Sweet-Parker scaling of reconnection, in the presence of turbulence we do not observe any dependence on Ohmic resistivity, confirming that the reconnection of weakly stochastic field is fast. We also do not observe a dependence on anomalous resistivity, which suggests that the presence of anomalous effects, e.g. Hall MHD effects, may be irrelevant for astrophysical systems with weakly stochastic magnetic fields.
\end{abstract}

Subject headings: galaxies: magnetic fields — physical processes: MHD — physical processes: turbulence - methods: numerical

\section{INTRODUCTION}

Magnetic fields play a key role in astrophysical processes such as star formation, the transport and acceleration of cosmic rays, accretion disks, solar phenomena, etc. (Crutcher 1999; Beck 2002; Schlickeiser 2004; Elmegreen \& Scalo 2004). Typically magnetic diffusion is very slow on astrophysical scales, so to a good approximation we can treat magnetic fields as being purely

Electronic address: kowal@astro.wisc.edu

Electronic address: lazarian@astro.wisc.edu

Electronic address: ethan@mcmaster.ca

Electronic address: otmian@astro.wisc.edu advected with the flow, which is frequently referred to in the literature as the "frozen in" condition for the plasma (see Moffat 1978).

Do we expect the frozen in condition to be violated in typical astrophysical conditions? The answer to this question is a qualified yes. Indeed, most astrophysical flows are chaotic; when adjacent parcels of fluids do not move in the same direction, the frozen-in magnetic fields become tangled. This can be easily visualized by viewing magnetic fields lines as threads moving with the fluid. When the distance between magnetic bundles of different direction becomes small, the finite resistivity of fluids 
starts to be important. In a generic situation of 3D flows, the bundles of magnetic fields come into contact with their neighbors at an angle of the order unity. Over the small scales at which fluid resistivity is important, magnetic field lines change their topology, or reconnect. However, once magnetic field energies become large, bending magnetic fields on small scales requires energies much larger than the turbulent energies on that scale. The magnetic field lines become stiff, even in the presence of strong turbulence, and the scales associated with contact between regions with very different magnetic fields become large.

What is the resulting reconnection speed? This is a vital question for many areas of astrophysics (see Biskamp 2000; Priest \& Forbes 2000; Bhattacharjee 2004; Zweibel \& Yamada 2009). A first guess could be that magnetic reconnection is generically slow in astrophysical circumstances. There is a large disparity in the scales involved. The scale of the magnetic flux bundle is astronomically large. The microphysical scale over which the Ohmic dissipation is important is relatively small. In this case the crossing magnetic bundles will create ubiquitous unresolved intersections or "knots" in the fluid. Magnetic tension that arises from those intersections as the magnetic bundles press against each other should dramatically change the properties of magnetized fluids ${ }^{1}$. This would be devastating news for most numerical MHD simulations, as numerical diffusion in the simulations is high and magnetic bundles in simulations easily reconnect.

To understand the difference between astrophysical reconnection and the one in numerical simulations, one should recall that the dimensionless combination that controls the reconnection rate is the Lundquist number $^{2}$, defined as $S=L V_{A} / \eta$, where $L$ is the length of the reconnection layer (see Figure 1. upper panel), $V_{A}$ is the Alfvén velocity, and $\eta$ is Ohmic diffusivity. Because of huge astrophysical sizes $L$ involved, the astrophysical Lundquist numbers are huge, e.g. for the ISM they are about $10^{16}$, while present-day MHD simulations correspond to $S<10^{4}$. As the numerical efforts scale as $L_{x}^{4}$, where $L_{x}$ is the size of the box, it is not feasible at present and will not be feasible in the foreseeable future to have simulations with the sufficiently high Lundquist numbers. Incidentally, this also presents a problem for numerical simulations of magnetic reconnection unless one has theoretically-derived scaling relations to test. Even with the limited resolution, numerical simulations are a good tool to study scaling relations, the point that has been proved by successful numerical studies of MHD turbulence.

Due to huge values of astrophysical Lundquist numbers, any rate of reconnection that depends on $S$ is extremely slow ${ }^{3}$. Fast reconnection is reconnection that does not depend on resistivity.

${ }^{1}$ Don Cox (private communication) refers to this state of entangled magnetic fields as astrophysical Jello or astrophysical felt, to reflect the peculiar non-fluid properties of the hypothetical substance.

2 The magnetic Reynolds number, which is the ratio of the magnetic field decay time to the eddy turnover time, is defined using the injection velocity $v_{l}$ as a characteristic speed instead of the Alfvén speed $V_{A}$, which is taken in the Lundquist number.

${ }^{3}$ The exception is the case when the dependence on $S$ is logarithmic.
What are the conditions that can make magnetic reconnection fast? Does it rely on special initial or boundary conditions for the flow, or does it require particular plasma effects? These are burning astrophysical questions, which, for example, define the extent that we can rely on numerical simulations of magnetized fluids as models of astrophysical phenomena. To understand processes of magnetic field generation associated with dynamos, the dynamics of the interstellar medium and accretion disks, or other related phenomena, it is important to understand magnetic reconnection. In most cases, astrophysical reconnection is difficult to observe, with the notable exception of solar flares (see Sturrock 1966; Masuda et al. 1994) and gamma ray bursts (see Galama et al. 1998). This sometimes creates an illusion that the importance of reconnection is limited to those selected phenomena.

A famous model of magnetic reconnection was suggested by Parker (1957) and Sweet (1958). Unfortunately, this model, usually referred to as Sweet-Parker reconnection, provides very slow reconnection speeds. In this model the reconnection rate is inversely proportional to the square root of the Lundquist number. This very slow speed comes from a geometrical constraint. The current sheet dividing two magnetized regions must be very thin in order for Ohmic resistivity to be important, but the plasma trapped in the current sheet must follow the local magnetic field lines in order to escape. A narrow current sheet implies a highly restricted outflow. Naturally, if the Lundquist number is large, e.g. $S=10^{16}$, the Sweet-Parker reconnection speed, $V_{S P} \approx V_{A} S^{-1 / 2}$, is negligible.

Fast reconnection has been investigated for many decades (see Biskamp 2000; Priest \& Forbes 2000, for reviews). In 1964 Petschek introduced the first fast magnetic reconnection model (see Petschek 1964). He proposed that extended magnetic bundles come into contact over a tiny area determined by the Ohmic diffusivity. This configuration, called an X-point configuration, differs dramatically from the expected generic configuration when magnetic bundles try to press their way through each other. Thus the first introduction of this model raised questions of dynamical self-consistency. An Xpoint configuration has to persist in the face of compressive bulk forces. However, numerical simulations have shown that an initial X-point configuration of magnetic field reconnection is unstable in the MHD limit for small values of the Ohmic diffusivity (Biskamp 1996) and the magnetic field will relax to a Sweet-Parker configuration. The physical explanation for this effect is simple. In the Petschek model shocks are required in order to maintain the geometry of the X-point. These shocks must persist and be supported by the flows driven by fast reconnection. The simulations showed that the shocks fade away and the contact region spontaneously increases.

More recently there has been progress in understanding the role of collisionless modes in stabilizing X-point reconnection. In particular, simulations using Hall MHD have produced stable X-point reconnection (Shay et al. 1998, 2004). This was the first numerical demonstration of fast reconnection, which raised the hope in astrophysical community that the problem of astrophysical reconnection can be solved. There is continuing discus- 
sion of whether this kind of fast reconnection persists at scales substantially larger than the ion inertial scale (see Bhattachariee et al. 2003) with a number of numerical studies (Wang et al. 2001; Smith et al. 2004; Fitzpatrick 2004) yielding reconnection rates that are not fast, but depend on resistivity. More importantly, one may also wonder to what extent Petscheck-type collisionless reconnection solves the problem of describing magnetic field dynamics in astrophysical settings. Apart from the issue of how natural it is to produce conditions that lead to Petscheck reconnection, one should note that the requirement that the upper limit on the collision rate required by this process is extremely restrictive. For instance, estimates in Yamada (2007) show that the scale of the reconnection current sheet should not exceed approximately 40 electron mean free paths. This is not satisfied in many astrophysical environments including the interstellar medium (Yamada 2007).

What happens in a collisional medium? Is reconnection in the interstellar medium slow? The latter, as mentioned above, would have catastrophic implications for the entire current crop of MHD simulations of interstellar processes.

Nearly simultaneously with the discovery of collisionless X-point reconnection, a model of magnetic reconnection in the presence of a weakly stochastic magnetic field was proposed by Lazarian \& Vishniac (1999, henceforth LV99). They claimed that the laminar magnetic fields considered in both Petscheck and Sweet-Parker reconnection models are exceptional in astrophysics. If we consider the interstellar medium as an example, a socalled "Big Power Law in the Sky" indicates the presence of turbulence on scales from tens of parsecs to thousands of kilometers (Armstrong et al. 1995). Thus, the fact that reconnection may be slow in a collisional fluid with laminar field lines does not necessarily imply that reconnection is generically slow. The model in LV99 predicted extended current sheets and wide outflows limited by the diffusive spread of magnetic field lines. In the limit of very weak turbulence or very large resistivities the model converges to the Sweet-Parker model.

For many years the quantitative predictions of the stochastic reconnection model in LV99 have not been tested explicitly. There is some implicit evidence in the favor of this model, e.g. observations of the thick reconnection current outflow regions observed in the Solar flares (Ciaravella \& Raymond 2008). Such outflow regions are incompatible with Petscheck reconnection. This is suggestive, but falls far short of constituting a definitive proof of the LV99 model.

It is, in general, extremely difficult to use analytic tools to test turbulent processes. However, Evink \& Aluie (2006) have studied the necessary conditions for reconnection in a turbulent conducting medium in the limit of infinitesimal resistivity. They list three separate necessary conditions, and prove that the flux conservation can be violated at an instant of time for an arbitrarily small scale, if at least one of the conditions is satisfied. The one that concerns us here is that there must be overlapping vortex and current sheets in the fluid. This is trivially satisfied if turbulent eddies on all scales give rise to current sheets with inflow/outflow properties of the kind we study here. While this shows that the LV99 model is consistent with the physical requirements for fast recon- nection, it does not prove that the model is viable.

Numerical simulations have provided the main testing ground for collisionless X-point reconnection. These simulations are typically performed in $2 \mathrm{D}$ with periodic boundary conditions and cannot be continued for longer than one dynamical time. This approach is inappropriate for stochastic reconnection where the goal is to obtain a stationary reconnection rate averaged over many dynamical times. For this reason we have followed Daughton et al. (2006) in using open outflow boundary conditions. Moreover, the LV99 model of reconnection is intrinsically three dimensional. A simplification, however, arises from the fact that LV99 predicts that fast reconnection takes place in the MHD approximation, making plasma effects irrelevant.

In this paper we describe the choice of boundary conditions as well as an appropriate measure of reconnection. Indeed, while the rate of reconnection can be trivially measured in the case of laminar magnetic fields, additional care is required in the estimation of the reconnection rate in the presence of a stochastic magnetic field.

In $₫ 2$ we review the LV99 model of reconnection and its theoretical predictions. In $\$ 3$ we describe in detail the numerical model we have built and studied in this paper. In $\$ 4$ we present methods used throughout the paper to measure the reconnection rate and we introduce a new, more general, method of estimating the reconnection rate which includes all processes contributing to the change of magnetic flux. In \$5 we present an extensive description of results obtained from studying our numeric model, which we discuss later in $\$ 6$, In $\$ 7$ we set forth our main conclusions.

\section{LAZARIAN-VISHNIAC (1999) MODEL}

We begin by reviewing the basic features of the stochastic reconnection model in Lazarian \& Vishniac (1999). This model might be seen as a generalization of the Sweet-Parker model (see Fig. 1) for the case of turbulent fields. Like the Sweet-Parker model it deals with an extremely generic configuration, which should arise naturally as magnetic flux tubes try to make their way one through another. This avoids the problems related to the preservation of outflow conditions which plague attempts to explain magnetic reconnection via Petscheck-type solutions. For example, if the outflow of reconnected flux and entrained matter is temporarily impeded through a fluctuation in the outflow, reconnection will slow down, but will not permanently change its nature.

The essential difference between the Sweet-Parker model and the model in LV99 is that while in the SweetParker model the outflow is limited by microphysical Ohmic diffusivity, in the LV99 model the large-scale magnetic field wandering determines the thickness of outflow. For extremely weak turbulence, when the range of magnetic field wandering becomes smaller than the width of the Sweet-Parker layer $L S^{-1 / 2}$, the reconnection rate reduces to the Sweet-Parker rate ${ }^{4}$.

\footnotetext{
${ }^{4}$ For the sake of simplicity, we do not raise here issues related to the stability of the Sweet-Parker current sheet. Estimates in LV99 suggest that laminar current sheets subject to tearing instabilities have reconnection rates which are just a bit faster than in the original Sweet-Parker model. These instabilities may, however, be important for increasing the 3D stochasticity of magnetic field lines and thus initiating fast reconnection. In this paper we do not address this effect either.
} 

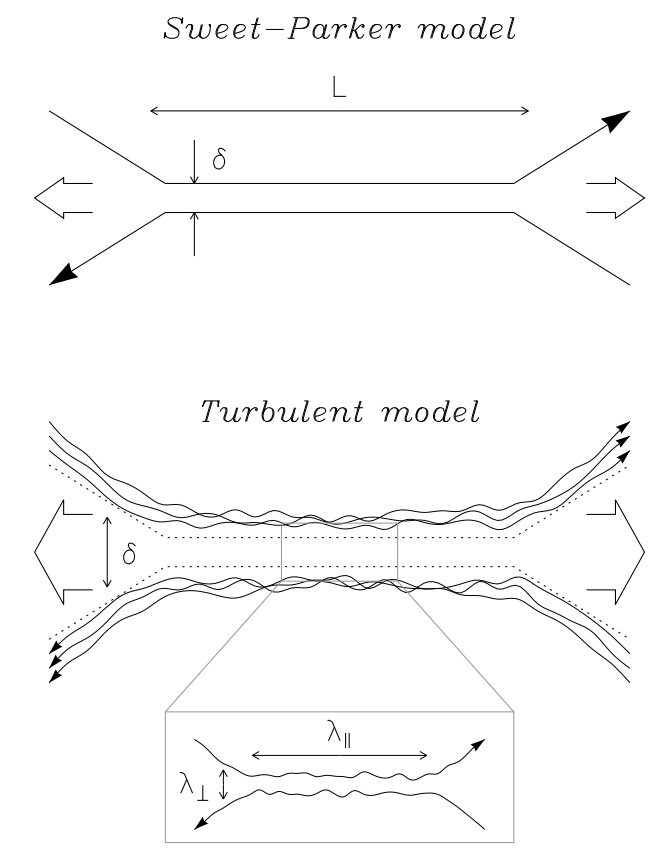

FIG. 1.- Upper plot: Sweet-Parker model of reconnection. The outflow is limited to a thin width $\delta$, which is determined by Ohmic diffusivity. The other scale is an astrophysical scale $L \gg \delta$. Magnetic field lines are assumed to be laminar. Middle plot: Turbulent reconnection model that accounts for the stochasticity of magnetic field lines. The stochasticity introduced by turbulence is weak and the direction of the mean field is clearly defined. The outflow is limited by the diffusion of magnetic field lines, which depends on macroscopic field line wandering rather than on microscales determined by resistivity. Low plot: An individual small scale reconnection region. The reconnection over small patches of magnetic field determines the local reconnection rate. The global reconnection rate is substantially larger as many independent patches reconnect simultaneously. Conservatively, the LV99 model assumes that the small scale events happen at a slow Sweet-Parker rate. Following Lazarian et al. (2004).

LV99 consider the case of a large scale, well-ordered magnetic field, of the kind that is normally used as a starting point for discussions of reconnection. In the presence of turbulence we anticipate that the field will have some small scale 'wandering'. On any given scale the typical angle by which field lines differ from their neighbors is $\phi \ll 1$, and this angle persists for a distance along the field lines $\lambda_{\|}$with a correlation distance $\lambda_{\perp}$ across field lines (see Fig. 1).

LV99 suggested that the presence of a random magnetic field component substantially enhances the reconnection rate, leading to fast reconnection. There are two phenomena mainly responsible for this:

- only a small fraction of any magnetic field line is subject to direct Ohmic annihilation. The fraction of magnetic energy that goes directly into heating the fluid drops down to zero as the fluid resistivity vanishes, and

- the presence of turbulence enables many magnetic field lines to enter the reconnection zone simultaneously.

In order to do quantitative estimates one has to adopt a model of MHD turbulence. Attempt to construct such a model can be traced back to Iroshnikov (1963) and Kraichnan (1965) papers as well as to later fundamental works (e.g. Montgomery \& Turner 1981; Shebalin et al. 1983; Higdon 1984, see also book by Biskamp 2003). A model for realistic compressible fluids may be constructed on the basis of the (Goldreich \& Sridhar 1995, henceforth GS95) model of incompressible turbulence ${ }^{5}$. Reasonable alternatives are expected to have only marginal impact on the nature of field line stochasticity (see also Cho \& Vishniac 2000; Maron \& Goldreich 2001; Lithwick \& Goldreich 2001; Cho et al. 2002; Cho \& Lazarian 2002, 2003), provided that the GS95 scalings are generalized to include the case of weak MHD turbulence. The equations in LV99 reflect the fact that when the turbulence is injected at subAlfvénic velocities, the cascading is initially weak and the perturbations can be reasonably well represented by a collection of Alfvén waves undergoing occasional interactions. However, the strength of the non-linear interactions increases as the perpendicular scale decreases and at the scale $l_{\perp}=l\left(v_{l} / V_{A}\right)^{2}$ the cascade becomes strong, i.e. with the GS95 critical balance between motions at parallel and perpendicular scales $l_{\perp} / v_{\perp} \approx l / V_{A}$ satisfied. The corresponding velocity at $l_{\perp}$ is $v_{\perp} \sim V_{A}\left(v_{l} / V_{A}\right)^{2}$ where $v_{l}$ is the velocity at which strong turbulence is being injected. For $v_{l} \ll V_{A}$ this scale may be small and the field wandering induced by the turbulence is reduced $^{6}$. The characteristics of the strong turbulent cascade dominate transport phenomena and predictions of reconnection speeds. In contrast the weak turbulent cascade has very little effect on field line stochasticity, since in this regime motions are largely periodic.

The modification of the global constraint induced by mass conservation in the presence of a stochastic magnetic field component is self-evident. Instead of being squeezed from a layer whose width is determined by Ohmic diffusion, the plasma diffuses through a much broader layer, $\delta \sim\left\langle y^{2}\right\rangle^{1 / 2}$ (see Fig. 1), determined by the diffusion of magnetic field lines. This suggests an upper limit on the reconnection speed of $\sim V_{A}\left(\left\langle y^{2}\right\rangle^{1 / 2} / L\right)$. This will be the actual speed of reconnection if the progress of reconnection in the current sheet does not impose a smaller limit. The value of $\left\langle y^{2}\right\rangle^{1 / 2}$ can be determined once a particular model of turbulence is adopted, but it is obvious from the very beginning that this value is determined by field wandering rather than Ohmic diffusion.

Following Lazarian et al. (2004, hereafter LVC04) we can generalize this upper limit to include the whole range of scales between the large scale eddy size and the current sheet thickness. Consider two points initially separated

5 The exact scalings of the MHD turbulence are still subject to debate and different corrections to the original GS95 scalings have been proposed to account for dynamical alignment, polarization intermittency and non-locality of the cascade (see Boldvrev 2005, 2006; Beresnvak \& Lazarian 2006, 2008b; Gogoberidze 2007). However, calculations in LV99 show that reconnection rates are only weakly dependent on the exact model of turbulence. Subtle refinements of the scalings that are currently debated can only be of marginal significance for this paper. For the sake of simplicity, we adopt in what follows the original GS95 scaling. We also do not consider imbalanced MHD turbulence (see Lithwick et al. 2007; Chandran 2008; Beresnyak \& Lazarian 2008a, 2009), although for the modest degrees of imbalance between the turbulent energy fluxes moving in the opposite directions, we do not expect substantial changes in our results

6 These comments ignore the compressible modes, but their role in field wandering is marginal anyhow (LV99). 
by the thickness of the current sheet. Translating both points along the field lines we can define their rms separation as a function of distance along the field lines, $\delta\left(\lambda_{\|}\right)$. This gives an upper limit on the local reconnection speed which is just $\sim\left(\delta / \lambda_{\|}\right) V_{A}$. However, the large scale current sheet contains many such reconnection regions, each involving the reconnection of independent field lines. In order to estimate the global reconnection rate we need to multiply each local reconnection rate with the number of simultaneous reconnection events happening on that scale. Taking $L / \lambda_{\|}$as our best estimate we get a global limit of

$$
V_{r e c} \leq \min \left[\frac{\delta\left(\lambda_{\|}\right)}{\lambda_{\|}} \frac{L}{\lambda_{\|}} V_{A}\right]
$$

which should be evaluated for all $\lambda_{\|}$between the length of the current sheet and the length of an individual piece of the current sheet such that translation along that piece will increase the rms separation by the thickness of the current sheet. As long as we are in the regime of strong turbulence this expression has a rather simple form. Each eddy scatters field lines by roughly its own width, so an eddy of parallel length $\lambda_{\|}$will have a corresponding $\delta \sim \lambda_{\perp}\left(\lambda_{\|}\right)$. With this in mind Eq. (1) becomes

$$
V_{\text {rec }} \leq \min \left[\frac{\lambda_{\perp} L}{\lambda_{\|}^{2}} V_{A}\right] .
$$

At its minimum Eq. (2) for GS95 model provides $V_{r e c}<$ $V_{A}$, which is a natural limit of the reconnection speed and not a constraint. In the Goldreich-Sridhar model of turbulence $\lambda_{\|} \propto \lambda_{\perp}^{2 / 3}$, so the minimum value of this expression corresponds to the largest scales. (Once $\lambda_{\|}$ exceeds the scale of the largest eddies we change models to a random walk with a fixed step size.)

This should make it clear why we are insensitive to the exact model of strong turbulence used in our calculation. In order to get a different answer we would need $\lambda_{\|} \propto \lambda_{\perp}^{1 / 2}$ or less, which is not supported by any of the available numerical evidence. On the other hand, how one models the weak turbulence does have an impact on the reconnection rate, since it will define the length of the largest scale eddies. Also, the presence of strong viscous damping can complicate our argument, by creating a laminar field line regime on very small scales and reducing $\delta$ far below its value in the strong turbulent regime. This point is discussed at length in LVC04 where it was argued that in some of the colder and denser phases of the interstellar medium we should expect a reduction in the reconnection speed by an order of magnitude or more. The numerical work presented here includes only a minimal amount of viscosity, so testing these arguments is beyond the scope of this paper.

The basic hypothesis of LV99 is that the the speed of magnetic reconnection in the presence of $3 \mathrm{D}$ stochasticity of magnetic field lines is given by the most stringent constraint imposed by the bottleneck condition expressed in Eq. (10) ${ }^{7}$. With the GS95 model of turbulence LV99

7 In LV99 other processes that can impede reconnection were found to be less restrictive. For instance, the tangle of reconnection field lines crossing the current sheet will need to reconnect repeatedly before individual flux elements can leave the current sheet behind. The rate at which this occurs can be estimated by obtained:

$$
V_{\text {rec }}=V_{A} \min \left[\left(\frac{L}{l}\right)^{1 / 2},\left(\frac{l}{L}\right)^{1 / 2}\right]\left(\frac{v_{l}}{V_{A}}\right)^{2},
$$

where $l$ and $v_{l}$ are the energy injection scale and turbulent velocity at this scale respectively. Note that the combination $V_{A}\left(v_{l} / V_{A}\right)^{2}$ is the velocity $v_{\text {trans }}$, i.e. the velocity at which the cascade transfers to the strong regime. This reflects the importance of strong turbulence for magnetic field wandering and magnetic reconnection. The term $(L / l)^{1 / 2}$ reflects the random walk which takes place for $L<l$ Evidently, the goal of our paper is to test formula (3).

There are several important characteristic features of Eq. (3). First, and most important, there is no dependence on resistivity. Second, in general we expect reconnection to be fast since in most cases the parameter ratios that enter the expression, i.e. the length of the reconnection layer $L$ divided by the injection scale $l$, and the injection velocity $v_{l}$ divided by the Alfvén velocity $V_{A}$ are of order unity. Finally, we note that in particular situations when turbulence is extremely weak the reconnection speed may be small.

Given the limited dynamical range of the simulations, we are forced to inject turbulent energy on scales less than $L$. Also, it is easier to control not $v_{l}$, but the energy injection power $P$. The power in the turbulent cascade is $P \sim v_{\text {turb }}^{2}\left(V_{A} / l\right)$ or $v_{l}^{4} /\left(l V_{A}\right)$, which reflects the fact that the turbulence is weak at the injection scale $l$ (see LV99). Using this in Eq. (3) we get

$$
V_{\text {rec }} \sim l P^{1 / 2},
$$

which is the prediction we will test here. In what follows we refer to the injection power and scale using $P_{i n j}$ and $l_{i n j}$, respectively.

\section{NUMERICAL SETUP}

\subsection{Governing Equations}

We use a higher-order shock-capturing Godunovtype scheme based on the essentially non oscillatory (ENO) spacial reconstruction and Runge-Kutta (RK) time integration (see Londrillo \& Del Zanna 2000; Del Zanna et al 2003, e.g.) to solve isothermal non-ideal MHD equations,

$$
\begin{aligned}
\frac{\partial \rho}{\partial t}+\nabla \cdot(\rho \boldsymbol{v}) & =0, \\
\frac{\partial \rho \boldsymbol{v}}{\partial t}+\nabla \cdot\left[\rho \boldsymbol{v} \boldsymbol{v}+\left(a^{2} \rho+\frac{B^{2}}{8 \pi}\right) I-\frac{1}{4 \pi} \boldsymbol{B} \boldsymbol{B}\right] & =\boldsymbol{f}, \\
\frac{\partial \boldsymbol{A}}{\partial t}+\boldsymbol{E} & =0,
\end{aligned}
$$

where $\rho$ and $\boldsymbol{v}$ are plasma density and velocity, respectively, $\boldsymbol{A}$ is the vector potential, $\boldsymbol{E}=-\boldsymbol{v} \times \boldsymbol{B}+\eta \boldsymbol{j}$ is the electric field, $\boldsymbol{B} \equiv \nabla \times \boldsymbol{A}$ is the magnetic field, $\boldsymbol{j}=\nabla \times \boldsymbol{B}$ is the current density, $a$ is the isothermal speed of sound, $\eta$ is the resistivity coefficient, and $f$ represents the forcing term. We used Harten-Lax-van Leer (HLL, Harten et al.

assuming that it constitutes the real bottleneck in reconnection events, and then analyzing each flux element reconnection as part of a self-similar system of such events. This turns out not to impede reconnection. 


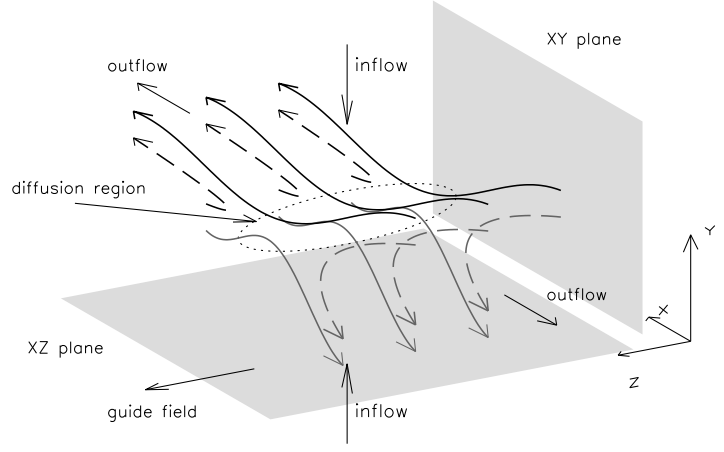

FIG. 2.- Schematic 3D visualization of the reconnection problem setup. Upper and lower parts of the domain contain incoming oppositely directed magnetic field lines (black and grey solid lines, respectively). The inflow bends the lines toward the center where they enter the diffusion region. In the diffusion region the lines reconnect and their product (dashed lines) is ejected along the $\mathrm{X}$ direction.

1983) and HLLD Riemann solvers (Mignone 2007) for solving the isothermal MHD equations. These schemes have widely different dissipation properties. The HLL solver was initially developed for solving Euler equations and later adopted for solution of general set of time dependent differential equations by averaging the Riemann fan over a region bound by the minimum and maximum local characteristic speeds. In this way one intermediate state was constructed. The HLLD Riemann solver takes into account the discontinuities resulting from the presence of magnetic field as well, separating this intermediate state into multiple intermediate states, resolving e.g. Alfvén waves with much less dissipation. In this paper we are considering the quasi-incompressible regime, where most of energy is transported by Alfvén waves, therefore the application of the HLLD solver seems to be a better choice. We incorporated the field interpolated constrained transport (CT) scheme based on a staggered mesh (see Londrillo \& Del Zanna 2000) into the integration of the induction equation (Eq. 7) to maintain the $\nabla \cdot \boldsymbol{B}=0$ constraint numerically.

Some selected simulations that we perform include anomalous resistivity modeled as

$$
\eta=\eta_{u}+\eta_{a}\left(\frac{|\boldsymbol{j}|}{j_{\text {crit }}}-1\right) H\left(\frac{|\boldsymbol{j}|}{j_{\text {crit }}}\right),
$$

where $\eta_{u}$ and $\eta_{a}$ describe uniform and anomalous resistivity coefficients, respectively, $j_{\text {crit }}$ is the critical level of the absolute value of current density above which the anomalous effects start to work, and $H$ is a step function. However, for most of our simulations $\eta_{a}=0$.

\subsection{Model Description and Initial Conditions}

Figures 2 and 3 show a 3D visualization and 2D projections of the reconnection problem setup. The domain contains two regions of oppositely directed magnetic field lines (see Fig. 2 and the left panel of Fig. 3). The incoming lines (solid lines in Fig. 2) are bent by the inflow $V_{i n}$ and enter the diffusion region. The diffusion region is characterized by the longitudinal scale $\Delta$ and its thickness $\delta$ (see the left panel of Fig. 3). The diffusion region extends along the full $\mathrm{Z}$ direction of the system. The incoming magnetic lines are not perfectly antiparallel. The projection of the magnetic topology on the XZ plane shows that the lines in the upper region (solid lines in the right panel of Fig. 3) and in the lower region (dashed lines) create an angle $\alpha$ determined by the strength of the shared component $B_{0 z}$. Once the incoming magnetic lines enter the diffusion region, they are reconnected and the product of this process is ejected along $\mathrm{X}$ direction with a speed $V_{\text {out }}$ (see Fig. 2 and the left panel of Fig. 3).

Our initial magnetic field is a Harris current sheet of the form $B_{x}(x, y, z)=B_{0 x} \tanh (y / \theta)$ initialized using the magnetic vector potential $A_{z}(x, y, z)=\ln |\cosh (y / \theta)|$. In addition, we use a uniform guide field $B_{z}(x, y, z)=$ $B_{0 z}=$ const. The initial setup is completed by setting the density profile from the condition of the uniform total (thermal plus magnetic) pressure $p_{T}(t=0, x, y, z)=$ const and setting the initial velocity to zero everywhere.

In order to initiate magnetic reconnection we add a small initial perturbation of vector potential $\delta A_{z}(x, y, z)=\delta B_{0 x} \cos (2 \pi x) \exp \left[-(y / d)^{2}\right]$ to the initial configuration of $A_{z}(t=0, x, y, z)$. Parameters $\delta B_{0 x}$ and $d$ describe the strength of the initial perturbation and thickness of the perturbed region, respectively.

We use dimensionless equations, so that the strength of the magnetic field is expressed in terms of the Alfvén velocity (defined by the antiparallel component of magnetic field) and the unperturbed density $\rho_{0}=1$. All other velocities are expressed as fractions of the fiducial Alfvén speed. The length of the box in X direction defines the unit of distance and time is measured in units of $L_{x} / V_{A}$. Initially, we set the strength of antiparallel magnetic field component $B_{0 x}$ to 1.0 and we vary the guide field $B_{0 z}$ between 0.0 and 1.0, which corresponds to the range of angle $\alpha \in\left(0^{\circ}, 90^{\circ}\right)$. For a particular run we use $B_{0 z}=4.0$, which corresponds to $\alpha \approx 152^{\circ}$. The speed of sound is set to 4.0 to suppress compressibility in the system. In order to study the importance of resistivity in the reconnection process we vary the resistivity coefficient $\eta_{u}$ between values $5 \cdot 10^{-4}$ and $5 \cdot 10^{-3}$ which are expressed in the dimensionless units. In the models where we include anomalous effects, we vary the anomalous resistivity coefficient $\eta_{a}$ between 0.0 and $2 \cdot 10^{-3}$. The parameters describing the initial perturbation are set to $\delta B_{0 x}=0.05$ and $d=0.1$.

\subsection{Boundary Conditions}

Our numerical model of LV99 reconnection evolves in a box with dimensions $L_{x}=L_{z}=1$ and $L_{y}=2$ with resolution $256 \times 512 \times 256$. It is extended in the $\mathrm{Y}$ direction in order to move the inflow boundaries far from the injection region. This minimizes the influence of the injected turbulence on the inflow.

We use three different types of boundary conditions in our models, each of them set along different direction: outflow boundary conditions along $\mathrm{X}$ direction, inflow boundary conditions along $\mathrm{Y}$ direction and periodic boundary conditions along $\mathrm{Z}$ direction.

We tested different types of open boundary conditions, including perfectly permeable wave-type boundary conditions derived from the linearized MHD equations similar to the method of characteristics (Hedstrom 1979), which seem to be the right choice. However, they are expensive since they require to track the linear MHD waves crossing the boundaries and they have a relatively small advantage over the simple "zero-gradient" bound- 

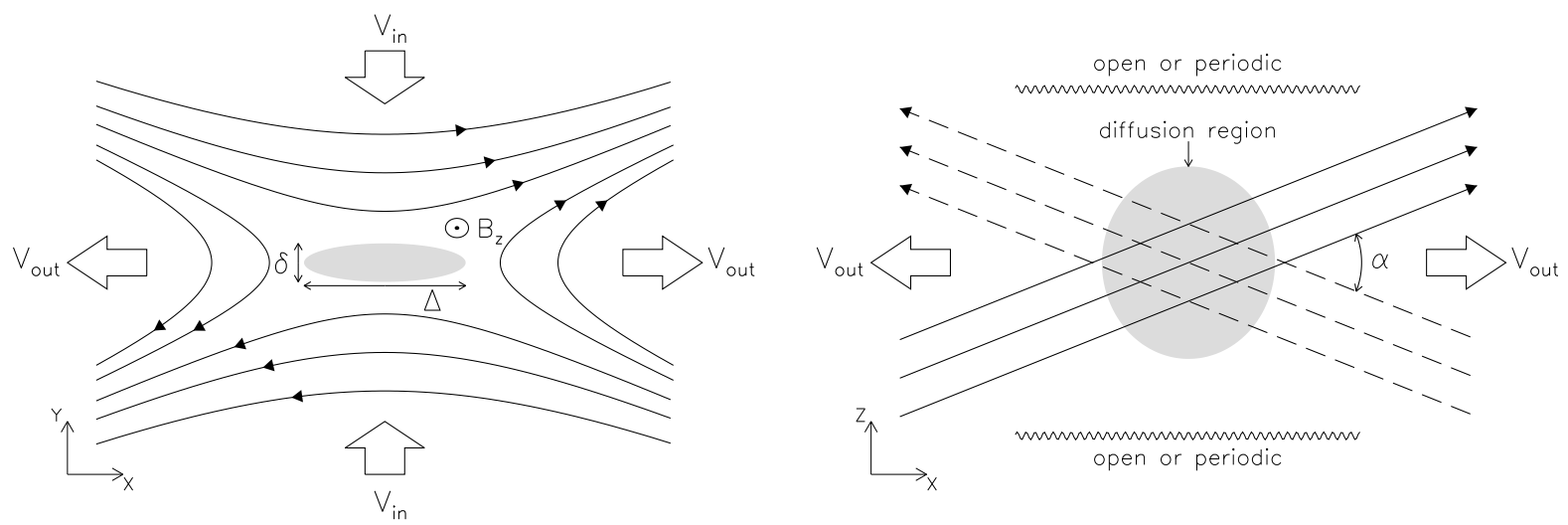

FIG. 3.- 3D magnetic field configuration in the studied problem projected on the XY (left) and XZ (right) planes. Left: XY projection of the magnetic field lines. The grey area describes the diffusion region where the incoming field lines reconnect. The longitudinal and transverse scales of the diffusion region are described by the parameters $\Delta$ and $\delta$, respectively. $B_{z}$ represents the direction of the guide field with respect to the plane of projection. We use inflow and outflow boundary conditions at X and Y directions, respectively. Right: XZ projection of the magnetic field lines as seen from the top. Solid and dashed lines show the incoming field lines from the upper and lower parts of the domain, respectively. We see that the oppositely directed field lines are not perfectly antiparallel but they create an angle $\alpha$ determined by the strength of the shared component $B_{z}$. The $\mathrm{Z}$ boundary conditions are open or periodic, depending on the model.

ary conditions in the application to our models, which we found the simplest and most robust for our purposes. In this type of open boundary conditions we set the normal derivatives of the fluid variables (density and momentum) to zero. This guarantees that all waves generated in the system are free to leave the box without significant reflections. In the presence of turbulence, however, this type of boundary does not enforce a constant mean value of density at the inflow boundary, which can result in a small loss of the total mass in the system. We will show later that the total mass loss is very small and does not influence our results.

As for the vector potential, we have tested Daughton's approach for open boundary conditions (see Daughton et al. 2006) in which the authors solved the properly set wave equations for each component. However, this approach was limited to the 2.5 dimensional case and its extension to the full 3D case requires a significant increase of complexity and, in our opinion, results in an unacceptably expensive method. Moreover, since Daughton et al. (2006) studied the problem of reconnection using a kinetic approach, they used the speed of light to evolve traveling electromagnetic waves. In the MHD approximation, this would imply a very large reduction in the time step, since the stability of the scheme depends on the Courant-Friedrichs-Lewy (CFL, Courant et al. 1928) condition, which in turn depends on the maximum speed in the system. This issue excluded this kind of approach from our consideration.

In the end we decided to use the simplest approach. We evolve the induction equation for the vector potential $\boldsymbol{A}$ based on a staggered mesh, which means that the vector potential components are located in the edge centers, i.e. $A_{x}(i, j+1 / 2, k+1 / 2), A_{y}(i+1 / 2, j, k+1 / 2)$, and $A_{z}(i+1 / 2, j+1 / 2, k)$, where $(i, j, k)$ describes the cell center position. Thus, using the method described in Londrillo \& Del Zanna (2000) we calculate magnetic field components located at the interface centers, $B_{x}(i+$ $1 / 2, j, k), B_{y}(i, j+1 / 2, k)$, and $B_{z}(i, j, k+1 / 2)$. These locations of collocation points for the vector potential completed by a CT integration of the induction equation guarantees the divergence-free evolution of magnetic field.
In the treatment of vector potential $\boldsymbol{A}$ at the boundary we set its components transverse to the considered boundary using the first order extrapolation, while the normal derivative of the normal component is set to zero. In this way the normal derivatives of transverse components of magnetic field are zero, while the normal component of magnetic field is calculated from the zerodivergence condition $\nabla \cdot \boldsymbol{B}=0$.

This approach prevents from the generation of divergence of $\boldsymbol{B}$ at the boundaries, however, it creates a small jump of the fluxes in the momentum equation across the boundary resulting from the presence of non-zero terms $\left(-B_{x}, B_{y}, B_{z}\right) \partial_{x} B_{x}$ at the $\mathrm{X}$ outflow boundary and $\left(B_{x},-B_{y}, B_{z}\right) \partial_{y} B_{y}$ at the $\mathrm{Y}$ inflow boundary. In order to justify the importance of these terms we have estimated directly from our models the velocity increment they produce at each time step. In models with the strongest turbulence the magnitudes of these terms were of order of $10^{-6}$ and $10^{-8}$ of $V_{A}$ at the $\mathrm{X}$ and $\mathrm{Y}$ boundaries, respectively, signifying their negligible importance in the presence of strong inflow and outflow which are of order of Alfvén speed in our models.

There is another constrain on the boundary conditions applying to the induction equations resulting from its non-ideality, i.e. the presence of resistive term and the treatment of current density $\boldsymbol{j}$ at the boundaries. When we set normal derivatives of transverse components of magnetic field to zero, we violate the continuity of current density. In the ideal case, $\eta=0$, the resistivity term is unimportant even if the current density is not continuous. However, once we introduce a non-zero resistivity coefficient, the resistive term $\eta \boldsymbol{j}$ in the induction equation becomes discontinuous at the boundary and we introduce a jump of electromotive force $\mathcal{E}=\boldsymbol{v} \times \boldsymbol{B}-\eta \boldsymbol{j}$ across the boundary. For example, considering the $\mathrm{Z}$ component of electromotive force $\mathcal{E}_{z}=v_{x} B_{y}-v_{y} B_{x}-\eta\left(\partial_{x} B_{y}-\partial_{y} B_{x}\right)$ we obtain a jump of $\mathcal{E}_{z}$ across the $\mathrm{X}$ boundary equal to $\Delta_{x} \mathcal{E}_{z}=\left(\mathcal{E}_{z}^{b}-\mathcal{E}_{z}^{d}\right)=\eta \partial_{x} B_{y}^{d}$, where indices $d$ (domain) and $b$ (boundary) stand for the limit values from the left and right sides of the considered boundary, respectively. This results in a constant generation of $B_{y}$ at the boundary if the second derivative $\partial_{x}^{2} B_{y}$ is not zero at that location. One way to remove this undesired effect is 
by setting the second order normal derivative of the field components to zero. However, there is no straightforward way to do this since it introduces another constraint on the boundary values and requires complex and expensive methods. We use another way of diminishing the importance of resistive terms and current density jump at the boundary. Instead of modifying the boundary conditions, we introduce a zone of decaying resistivity close to the boundary. This means that the resistivity is constant in most of the computational domain. Only in a thin zone near the boundary, the value of resistivity $\eta_{u}$ decays down to a very small value, which is estimated to be around the numerical resistivity $\eta_{n}$ of our code. In our models we adopt the value of $\eta_{n}=3 \cdot 10^{-4}$. Naturally, by introducing such resistivity we do not change the reconnection speeds. There are two advantages to this approach. First, the outflow in our models is not constrained by the jump of resistive terms growing with the increasing value of resistivity $\eta_{u}$, and is determined solely by the evolution in the central part of the domain, where the resistivity is constant and uniform. Second, we control the importance of numerical effects by introducing a small, but larger than numerical resistivity value of $\eta_{n}$ at the boundary. The validation of this method is presented in $\$ 5.2 .4$.

The above boundary conditions represent open boundaries, which adjust during the evolution of the system. This means that we do not set fixed values of the fluid and magnetic variables and do not drive the flow at the boundaries in order to achieve a stationary reconnection process in the system.

\subsection{Model of Turbulence}

In our models we drive turbulence using a method described by Alvelius (1999). The forcing is implemented in spectral space where it is concentrated around a wave vector $k_{i n j}$ corresponding to the injection scale $l_{i n j}$. We perturb a number $N_{f}$ of discrete Fourier components of velocity in a shell extending from $k_{i n j}-\Delta k_{i n j}$ to $k_{i n j}+\Delta k_{i n j}$ with a Gaussian profile of the half width $k_{c}$ and the peak amplitude $\tilde{v}_{f}$ at the injection scale (see Tab. 1 for exact values in all models). In all models $k_{c}=0.4$. Since we can control the scale of injection, the power input is introduced into the flow at an arbitrary scale. The amplitude of driving is solely determined by its power $P_{i n j}$, the number of driven Fourier components and the time step of driving $\Delta t_{f}$, which in2 all our models is equal to $10^{-5}$. The parameters describing our forcing do not change during the evolution of the system. Because we perturb discrete number $N_{f}$ of Fourier components which depends on the injection scale $k_{i n j}$ and the thickness of perturbed shell $\Delta k_{i n j}$, the amplitude $\tilde{v}_{f}$ varies with the injection scale in order to keep the same power input $P_{i n j}$. In our models $\tilde{v}_{f}$ is always a small fraction of Alfvén speed (see Tab. 1).

The randomness in time makes the force neutral in the sense that it does not directly correlate with any of the time scales of the turbulent flow, and it also determines the power input solely by the force-force correlation. This means that it is possible to generate different desirable turbulence states, such as axisymmetric turbulence, where the degree of anisotropy of the forcing can be chosen a priori through the forcing parameters. In the models presented in this paper we use isotropic forcing only.

In particular, the total amount of power input from the forcing can be set to balance a desired dissipation at a statistically stationary state. In order to contribute to the input power in the discrete equations from the forceforce correlation only, the force is determined so that the velocity-force correlation vanishes for each Fourier mode. The procedure of reducing the velocity-force correlation is described in Alvelius (1999).

We drive turbulence in a subvolume of the domain. The size of the subvolume is determined by two scales, the radius $r_{d}$ on the $\mathrm{XZ}$ plane around the center of the domain and the height $h_{d}$ describing the thickness of the driving region from the midplane. In this way we avoid driving turbulence at the boundary and reduce the influence of driving on the inflow or outflow. All models are evolved without turbulence for several dynamical times in order to allow the system to achieve laminar stationary reconnection. Then, at a given time $t_{b}$ we start driving turbulence, increasing its amplitude to the desired level, until time $t_{e}$. In this way we let the system to adjust to a new state. From time $t_{e}$ the turbulence is driven with the full power $P_{i n j}$.

On the right hand side of Eq. (6), the forcing is represented by a function $\boldsymbol{f}=\rho \boldsymbol{a}$, where $\rho$ is local density and $\boldsymbol{a}$ is random acceleration calculated using the method described above.

We do not set the viscosity coefficient explicitly in our models. The scale at which the dissipation starts to be important is defined by the numerical diffusivity of the scheme. The ENO-type schemes are considered relatively low diffusion (see Liu \& Osher 1998; Levy et al. 1999, e.g.). The numerical diffusion depends not only on the adopted numerical scheme but also on the "smoothness" of the solution, so it changes locally in the system. In addition, it is also a time-varying quantity. All these problems make its estimation difficult and incomparable between different applications. However, the dissipation scales can be estimated approximately from the velocity spectra. Supported by our studies of turbulence (see Kowal et al 2007; Kowal \& Lazarian 2007) where we used similar code, we estimated the dissipation scale $k_{\nu} \approx 30$ for the resolution of models presented here.

\subsection{Table of Simulated Models}

In Table 1 we list parameters of all the models presented in this paper. We divided them into several groups. In each group we calculated models in order to study the dependence of the reconnection rate on a characteristic parameter of turbulence or resistivity. We have studied the dependence of reconnection on the power of turbulence (models "PD"), injection scale (models "SD"), uniform resistivity (models "RD"), anomalous resistivity (models "AD"), and dependence on the guide field (models "BD").

Among all parameters of the model we list those which vary, i.e. the strength of guide field $B_{0 z}$, the uniform and anomalous resistivities, $\eta_{u}$ and $\eta_{a}$, respectively, the power of turbulence $P_{i n j}$ and its injection scale $k_{i n j}$ with the half-thickness of the injection shell $\Delta k_{i n j}$, the number of perturbed Fourier components of velocity $N_{f}$ and the amplitude of perturbation $\tilde{v}_{f}$ at the injection scale. In addition, we include some of the most important parameters obtained from the simulations, such as the 
TABLE 1

LIST OF MODELS.

\begin{tabular}{|c|c|c|c|c|c|c|c|c|c|c|c|}
\hline Name & $B_{0 z}$ & $\eta_{u}\left[10^{-3}\right]$ & $\eta_{a}\left[10^{-3}\right]$ & $P_{i n j}$ & $k_{i n j}$ & $\Delta k_{i n j}$ & $N_{f}$ & $\tilde{v}_{f}$ & $v_{l}(\mathrm{t}=12)$ & $\tilde{V}_{A}$ & $\beta \equiv p / p_{m a g}$ \\
\hline \multirow[t]{10}{*}{$\mathrm{PD}$} & 0.1 & 1.0 & 0.0 & 0.1 & 8 & 0.5 & 96 & 0.0015 & 0.031 & 1.05 & 31.7 \\
\hline & 0.1 & 1.0 & 0.0 & 0.2 & 8 & 0.5 & 96 & 0.0022 & 0.041 & 1.05 & 31.7 \\
\hline & 0.1 & 1.0 & 0.0 & 0.5 & 8 & 0.5 & 96 & 0.0035 & 0.051 & 1.05 & 31.7 \\
\hline & 0.1 & 1.0 & 0.0 & 1.0 & 8 & 0.5 & 96 & 0.0049 & 0.065 & 1.05 & 31.7 \\
\hline & 0.1 & 1.0 & 0.0 & 2.0 & 8 & 0.5 & 96 & 0.0069 & 0.084 & 1.05 & 31.7 \\
\hline & 1.0 & 1.0 & 0.0 & 0.1 & 8 & 0.5 & 96 & 0.0015 & 0.042 & 1.41 & 16.0 \\
\hline & 1.0 & 1.0 & 0.0 & 0.2 & 8 & 0.5 & 96 & 0.0022 & 0.043 & 1.41 & 16.0 \\
\hline & 1.0 & 1.0 & 0.0 & 0.5 & 8 & 0.5 & 96 & 0.0035 & 0.056 & 1.41 & 16.0 \\
\hline & 1.0 & 1.0 & 0.0 & 1.0 & 8 & 0.5 & 96 & 0.0049 & 0.071 & 1.41 & 16.0 \\
\hline & 1.0 & 1.0 & 0.0 & 2.0 & 8 & 0.5 & 96 & 0.0069 & 0.083 & 1.41 & 16.0 \\
\hline \multirow[t]{10}{*}{ SD } & 0.1 & 1.0 & 0.0 & 1.0 & 5 & 0.5 & 24 & 0.0028 & 0.092 & 1.05 & 31.7 \\
\hline & 0.1 & 1.0 & 0.0 & 1.0 & 8 & 0.5 & 96 & 0.0049 & 0.063 & 1.05 & 31.7 \\
\hline & 0.1 & 1.0 & 0.0 & 1.0 & 12 & 1.0 & 48 & 0.0018 & 0.059 & 1.05 & 31.7 \\
\hline & 0.1 & 1.0 & 0.0 & 1.0 & 16 & 1.0 & 96 & 0.0049 & 0.035 & 1.05 & 31.7 \\
\hline & 0.1 & 1.0 & 0.0 & 1.0 & 25 & 1.0 & 265 & 0.0014 & 0.030 & 1.05 & 31.7 \\
\hline & 1.0 & 1.0 & 0.0 & 1.0 & 5 & 0.5 & 24 & 0.0028 & 0.092 & 1.41 & 16.0 \\
\hline & 1.0 & 1.0 & 0.0 & 1.0 & 8 & 0.5 & 96 & 0.0049 & 0.068 & 1.41 & 16.0 \\
\hline & 1.0 & 1.0 & 0.0 & 1.0 & 12 & 1.0 & 48 & 0.0018 & 0.059 & 1.41 & 16.0 \\
\hline & 1.0 & 1.0 & 0.0 & 1.0 & 16 & 1.0 & 96 & 0.0049 & 0.034 & 1.41 & 16.0 \\
\hline & 1.0 & 1.0 & 0.0 & 1.0 & 25 & 1.0 & 265 & 0.0014 & 0.027 & 1.41 & 16.0 \\
\hline \multirow[t]{8}{*}{$\mathrm{RD}$} & 0.1 & 0.5 & 0.0 & 1.0 & 8 & 0.5 & 96 & 0.0049 & 0.066 & 1.05 & 31.7 \\
\hline & 0.1 & 1.0 & 0.0 & 1.0 & 8 & 0.5 & 96 & 0.0049 & 0.065 & 1.05 & 31.7 \\
\hline & 0.1 & 2.0 & 0.0 & 1.0 & 8 & 0.5 & 96 & 0.0049 & 0.067 & 1.05 & 31.7 \\
\hline & 0.1 & 4.0 & 0.0 & 1.0 & 8 & 0.5 & 96 & 0.0049 & 0.066 & 1.05 & 31.7 \\
\hline & 0.1 & 5.0 & 0.0 & 1.0 & 8 & 0.5 & 96 & 0.0049 & 0.065 & 1.05 & 31.7 \\
\hline & 1.0 & 1.0 & 0.0 & 1.0 & 8 & 0.5 & 96 & 0.0049 & 0.068 & 1.41 & 16.0 \\
\hline & 1.0 & 2.0 & 0.0 & 1.0 & 8 & 0.5 & 96 & 0.0049 & 0.067 & 1.41 & 16.0 \\
\hline & 1.0 & 4.0 & 0.0 & 1.0 & 8 & 0.5 & 96 & 0.0049 & 0.067 & 1.41 & 16.0 \\
\hline \multirow[t]{4}{*}{$\overline{A D}$} & 0.2 & 0.5 & 0.0 & 0.5 & 5 & 0.5 & 24 & 0.0020 & 0.051 & 1.10 & 30.8 \\
\hline & 0.2 & 0.5 & 0.5 & 0.5 & 5 & 0.5 & 24 & 0.0020 & 0.051 & 1.10 & 30.8 \\
\hline & 0.2 & 0.5 & 1.0 & 0.5 & 5 & 0.5 & 24 & 0.0020 & 0.051 & 1.10 & 30.8 \\
\hline & 0.2 & 0.5 & 2.0 & 0.5 & 5 & 0.5 & 24 & 0.0020 & 0.051 & 1.10 & 30.8 \\
\hline \multirow[t]{4}{*}{$\mathrm{BD}$} & 0.1 & 1.0 & 0.0 & 1.0 & 8 & 0.5 & 96 & 0.0049 & 0.065 & 1.05 & 31.7 \\
\hline & 0.5 & 1.0 & 0.0 & 1.0 & 8 & 0.5 & 96 & 0.0049 & 0.066 & 1.22 & 25.6 \\
\hline & 1.0 & 1.0 & 0.0 & 1.0 & 8 & 0.5 & 96 & 0.0049 & 0.068 & 1.41 & 16.0 \\
\hline & 4.0 & 1.0 & 0.0 & 1.0 & 8 & 0.5 & 96 & 0.0049 & 0.067 & 2.24 & 1.9 \\
\hline
\end{tabular}

peak amplitude at the injection scale $v_{l}$ obtained from the spectra of velocity at the final time of simulation $(\mathrm{t}=12.0)$, the total Alfvén speed $\tilde{V}_{A}=\sqrt{\left(B_{0 x}^{2}+B_{0 z}^{2}\right) / \rho_{0}}$ and the plasma beta parameter $\beta=p / p_{\text {mag }}$. Note that the Alfvén speed $V_{A}=\left|B_{0 x}\right| / \sqrt{\rho_{0}}$ defined by the strength of the antiparallel component of magnetic field is the same for all models and equals 1.0.

All models presented in this section were calculated with the grid size $\Delta x \approx 0.004$ corresponding to the resolution $256 \times 512 \times 256$. In the process of constructing our numerical model we have performed number of tests for convergence of numerical solution during the SweetParker stage. These studies indicated that the convergence is reached for both HLL and HLLD solvers at this resolution, but the HLL scheme characterizes by a much higher numerical dissipation. This is expected since the HLL solver smooths out the rotational discontinuities resulting from the presence of magnetic field. As will will show in $\S 5.1$, the numerical resistivity in the models calculated with the HLLD scheme has the value of about $6.1 \cdot 10^{-4}$. The convergence studies signify that the same models calculated using the HLL scheme with the same resolution reveal a higher numerical resistivity of order of $8.7 \cdot 10^{-4}$, which is over $40 \%$ larger than $\eta_{\text {num }}$ in the HLLD scheme for the same grid size $\Delta x \approx 0.004$. For models with lower resolution where the grid size $\Delta x \approx 0.008$ we obtained values of the numerical resistivity $\eta_{\text {num }}^{\text {hlld }} \approx 6.6 \cdot 10^{-4}$ and $\eta_{\text {num }}^{\text {hll }} \approx 1.12 \cdot 10^{-3}$ for the HLLD and HLL schemes, respectively. Here, the difference of numerical resistivity between both resolutions for the HLLD solver is insignificant, justifying the convergence of the solution, while the HLL scheme behaves rather poorly showing an increase of numerical dissipation by over $75 \%$ with respect to the model with smaller grid size. Thus all our models presented here were calculated using the HLLD scheme if not indicated otherwise.

\section{RECONNECTION RATE MEASURE}

We measure the reconnection rate by averaging the inflow velocity $V_{i n}$ divided by the Alfvén speed $V_{A}$ over the inflow boundaries, i.e.

$$
\left\langle V_{i n} / V_{A}\right\rangle=\frac{1}{2} \int_{S} d x d z\left(\left.\frac{v_{y}}{V_{A}}\right|_{y=y_{\min }}-\left.\frac{v_{y}}{V_{A}}\right|_{y=y_{\max }}\right)
$$

where $S$ defines the area of the XZ inflow boundaries. Since we have two XZ boundaries, located at $y=y_{\min }$ and $y=y_{\max }$, we need to take half of the resulting integral. This measure works well for laminar reconnection, when the system is perfectly stable and where the time derivative of the magnetic flux is zero. In the presence of turbulence, however, this time derivative can fluctuate or the turbulence in the center of the box could affect the flow of the plasma. In this way we would get a flow of magnetic flux without the presence of reconnection. In order to include all effects contributing to the change of 


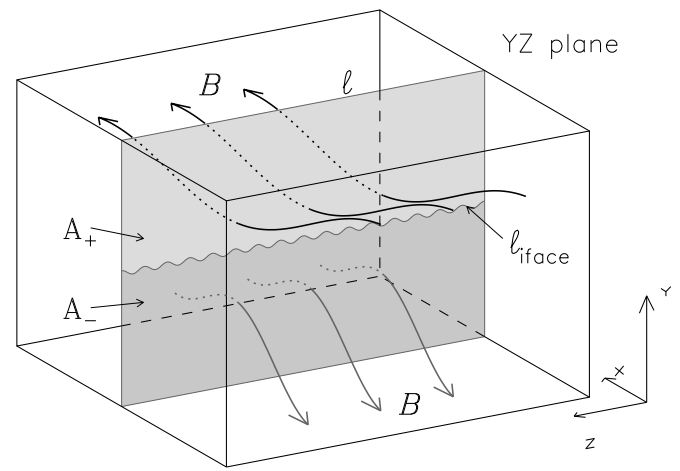

FIG. 4.- Schematic 3D visualization of the reconnection rate evaluation. $A_{+}$and $A_{-}$areas are defined by the sign of $B_{x}$ component.

magnetic flux, we define a new more general measure of the reconnection rate.

We start by considering a conserved quantity; the magnetic flux $\Phi$. First, we consider the flux contained within a plane inside the simulation volume (see Fig. 4). If $\hat{x}$ is the direction of the reconnecting field, then we start by considering the time derivative of the net flux of $B_{x}$. It is

$$
\partial_{t} \Phi=-\oint \boldsymbol{E} \cdot d \boldsymbol{l}=\oint(\boldsymbol{v} \times \boldsymbol{B}-\eta \boldsymbol{j}) \cdot d \boldsymbol{l}
$$

This equation is not exactly satisfied in our simulations unless we allow for numerical resistivity. This discrepancy can be used to derive an independent measure of the effective resistivity of the code, which is roughly consistent with value derived from the speed of laminar reconnection. Now we split the area of integration into two pieces, $A_{+}$and $A_{-}$, defined by the sign of $B_{x}$ (see Fig. 4). Instead of adding two areas we subtract them, i.e.

$$
\partial_{t} \Phi_{+}-\partial_{t} \Phi_{-}=\partial_{t} \int\left|B_{x}\right| d A
$$

which we can write explicitly in terms of line integrals around $A_{+}$and $A_{-}$

$$
\begin{aligned}
\partial_{t} \int\left|B_{x}\right| d A & =\oint \boldsymbol{E} \cdot d \boldsymbol{l}_{+}-\oint \boldsymbol{E} \cdot d \boldsymbol{l}_{-} \\
& =\oint \operatorname{sign}\left(B_{x}\right) \boldsymbol{E} \cdot d \boldsymbol{l}+\int 2 \boldsymbol{E} \cdot d \boldsymbol{l}_{\text {iface }}
\end{aligned}
$$

where $\boldsymbol{l}_{\text {iface }}$ is the line separating $A_{+}$and $A_{-}$(see Fig. 4). The last term describes the mutual annihilation of positive and negative $B_{x}$ along the line separating them and by definition, this is the reconnection rate. Note that this includes the motion of already reconnected flux lines through the plane of integration. Rather than try to calculate it numerically, we define the interface term as $-2 V_{r e c}\left|B_{x, \infty}\right| L_{z}$, where $\left|B_{x, \infty}\right|$ is the asymptotic absolute value of $B_{x}$, and $L_{z}$ is the width of the box. We can then calculate the other terms which do not involve trying to find the interface and the parallel component of the electric field. The end result, which is the new measure of reconnection rate, is

$$
V_{\mathrm{rec}}=\frac{1}{2\left|B_{x, \infty}\right| L_{z}}\left[\oint \operatorname{sign}\left(B_{x}\right) \boldsymbol{E} \cdot d \boldsymbol{l}-\partial_{t} \int\left|B_{x}\right| d A\right]
$$

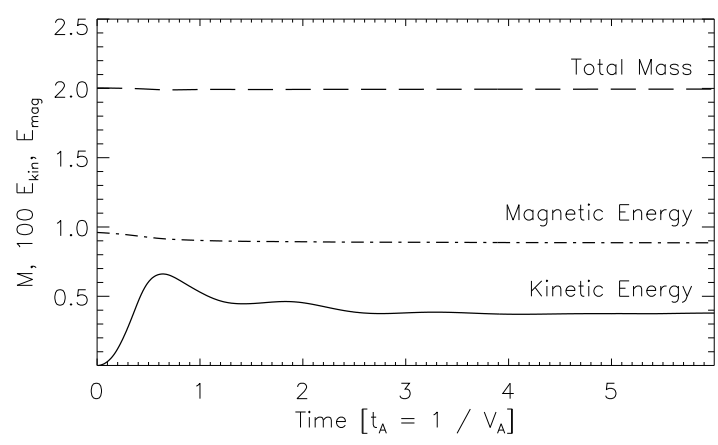

Fig. 5.- Evolution of the total mass $M$, and kinetic and magnetic energies, $E_{k i n}$ and $E_{m a g}$, respectively, during the SweetParker stage. The kinetic energy $E_{k i n}$ has been amplified by a factor of 100 to visualize its evolution more clearly. The resistivity in this model is set to $\eta_{u}=10^{-3}$ and the guide field $B_{0 z}=0.1$.

The electric field $\boldsymbol{v} \times \boldsymbol{B}-\eta \boldsymbol{j}$ can be further divided into an advection term $\boldsymbol{v} \times B_{x} \hat{x}$, a shear term $\boldsymbol{v} \times\left(B_{y} \hat{y}+B_{z} \hat{z}\right)$, and a resistive term $-\eta \boldsymbol{j}$. With this in mind the line integral can be rewritten as

$$
\begin{array}{r}
\oint \operatorname{sign}\left(B_{x}\right) \boldsymbol{E} \cdot d \boldsymbol{l}=\oint\left|B_{x}\right|\left(\boldsymbol{v}_{\perp} \times \hat{x}\right) \cdot d \boldsymbol{l} \\
+\oint \operatorname{sign}\left(B_{x}\right) v_{x}\left(\hat{x} \times \boldsymbol{B}_{\perp}\right) \cdot d \boldsymbol{l}-\oint \eta \boldsymbol{j} \cdot d \boldsymbol{l} .
\end{array}
$$

This new reconnection measure contains the time derivative of the absolute value of $B_{x}$, and a number of boundary terms, such as advection of $B_{x}$ across the boundary and the boundary integral of the resistive term $\eta \boldsymbol{j}$. The additional terms include all processes contributing the time change of $\left|B_{x}\right|$. In particular, they can have non-zero values.

\section{RESULTS}

In this section we describe the results obtained from our three dimensional simulations of magnetic reconnection in the presence of turbulence. First, we investigate Sweet-Parker reconnection, the stage before we inject turbulence. A full understanding of this stage is required in order to perform further analysis of reconnection in the presence of turbulence.

\subsection{Sweet-Parker Reconnection}

As we described in $\$ 3.2$. Sweet-Parker reconnection develops in our models as a result of an initial vector potential perturbation. In order to reliably study the influence of turbulence on the evolution of such systems, we need to reach the stationary Sweet-Parker reconnection before we start injecting energy.

Figure 5 shows the evolution of total mass, kinetic and magnetic energies until we start injecting the turbulence (i.e. $t=7$ ). All quantities, after some initial adaptation, reach almost constant values. We remind the reader, that the system evolves in the presence of open boundary conditions, which do not guarantee perfect conservation of mass and total energy. Nevertheless, conservation of these quantities is well satisfied during the Sweet-Parker stage in our models.

The reconnection rate, shown in Figure 6 (solid thick line representing the new reconnection rate measure), also confirms that we have reached a stationary state. Initially, the reconnection rate $V_{\text {rec }}$ grows until time 


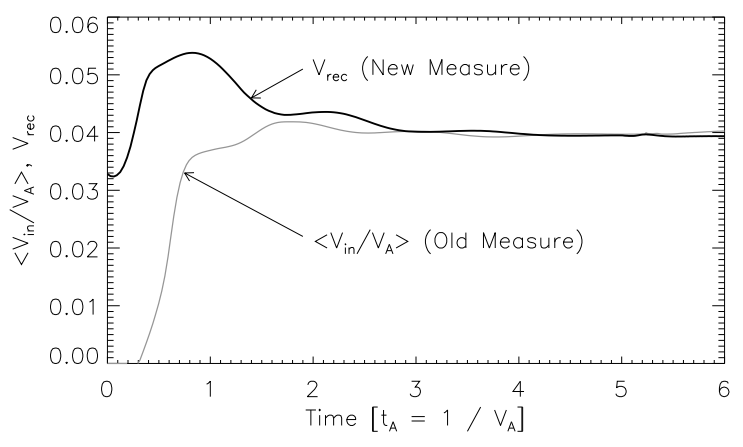

Fig. 6.- Evolution of the reconnection rates, $\left\langle V_{i n} / V_{A}\right\rangle$ (old) and $V_{\text {rec }}$ (new), for the same model as in Fig. 5 The reconnection rate grows initially until it reaches the stationary solution. Note, that both reconnection measures coincide during the later evolution.

$t \approx 1.0$, when it reaches a maximum value of $\approx 0.05$. Later on, it drops a bit approaching a value of 0.04. During the last period of about three Alfvén time units, the change of the reconnection rate is very small. We assume that these conditions guarantee a nearly steady state evolution of the system. In this plot we also show the evolution of old reconnection measure $\left\langle V_{i n} / V_{A}\right\rangle$ which do not include time variation of the flux. We see, that after the initial discrepancy they coincide and show the same value of reconnection speed.

In order to check the reliability of our code, we estimate characteristic parameters of the laminar reconnection, such as thickness of current sheet $\delta$, and the inflow and outflow speeds, $V_{\text {in }}$ and $V_{\text {out }}$, respectively (see Fig. 7], from the profiles of the absolute value of current density $|\boldsymbol{j}|$ along the $\mathrm{Y}$ direction and the profiles of $v_{x}$ and $v_{y}$ along the $\mathrm{X}$ and $\mathrm{Y}$ directions, respectively. We compare these estimates to the values obtained from the equations describing Sweet-Parker model. We limit this procedure to one model with a resolution of $256 \times 512 \times 256$, uniform resistivity $\eta_{u}=10^{-3}$ and shared component of magnetic field $B_{0 z}=0.1$. We analyze the snapshot obtained at the time $t=7$ before we start introducing turbulence.

First, using the Ohmic dissipation formula $V_{i n}=$ $\eta / \delta$ we estimate the total resistivity coefficient including the numerical contribution. We obtain a value of $\eta=\eta_{u}+\eta_{\text {num }}=V_{\text {in }} \delta \approx 1.64 \cdot 10^{-3}$. It indicates that the numerical resistivity $\eta_{\text {num }}$ has a value of about $60 \%$ of the explicit uniform resistivity. We use this value of resistivity in the subsequent estimates. Next, using mass conservation, $V_{\text {in }} L=V_{\text {out }} \delta$ (Parker 1957; Sweet 1958), we estimate the outflow speed $V_{\text {out }}$. Since the size of our box along $\mathrm{X}$ direction is $L=1.0$, this gives a value of $V_{\text {out }} \approx 0.394$, which agrees well with the value $V_{\text {out }}=0.395$ obtained from the profile of $v_{x}$. Since we already know the characteristic parameters $V_{\text {out }}, L$, and $\eta$, we can estimate the Lundquist number $S \equiv L V_{\text {out }} / \eta$ for our models. Substituting estimated $V_{\text {out }}, L$, and $\eta$ we obtain a value of $S \approx 236$. As expected, our numerical models are far from the conditions observed in typical astrophysical objects where $S \sim 10^{10}-10^{20}$. Nevertheless, we can still use them to determine their dependence on the characteristics of the environment. From $V_{\text {out }}$ and $S$ we can estimate the Sweet-Parker reconnection rate $V_{\text {rec }}=V_{\text {out }} S^{-1 / 2}$, which gives a value of $\sim 0.0251$, matching almost perfectly the value of $V_{\text {in }}$ fit from the model. These estimates indicate, that our nu-
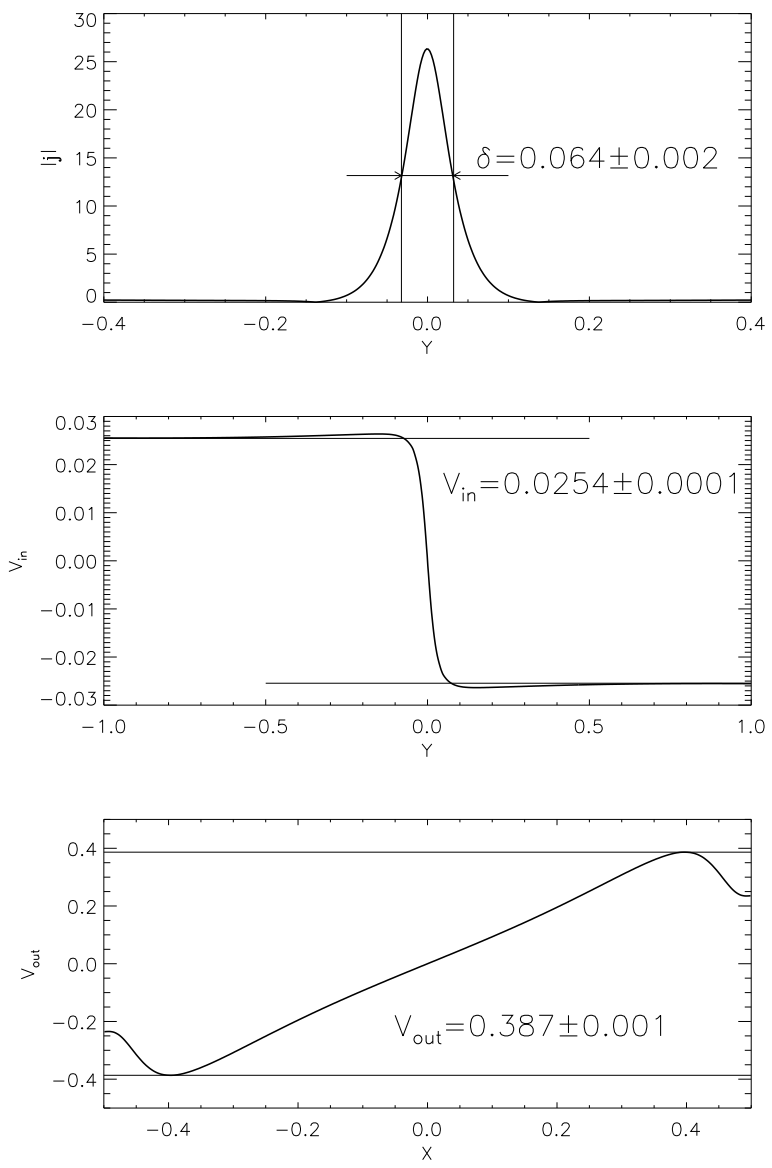

FIG. 7.- Estimates of the current sheet thickness $\delta$ (top), the inflow (middle) and outflow (bottom) speeds $V_{\text {in }}$ and $V_{\text {out }}$, respectively at the time $t=7$ for the model with $\eta_{u}=10^{-3}$ and $B_{0 z}=0.1$.

merical model exhibits Sweet-Parker reconnection during the stationary phase.

Before presenting our results in the presence of turbulence, we describe the topology of the flow and magnetic field in the laminar case. In Figure 10 we present the velocity and magnetic field configuration of the steady state. In the left panel we show the topology of the velocity field as textures. The brightness of texture corresponds to the amplitude of the field, while the texture itself shows the direction of the field lines. The topology of the velocity field is mainly characterized by strong outflow regions along the midplane. This outflow is produced by the constant reconnection process at the diffusion region near the center and the ejection of the reconnected magnetic flux through the left and right $\mathrm{X}$ boundaries. The system is in a steady state when the flux, which reconnects, is counterbalanced by the incoming unreconnected flux. The inflow is much slower than the outflow, but its direction is still apparent in the left plot of Figure 10.

The topology of the magnetic field is shown in the middle panel of Figure 10. We recognize the antiparallel configuration of the magnetic lines with uniform strength out of the midplane region. Near the midplane, the horizontal magnetic lines are reconnected generating the $\mathrm{Y}$ component, which is ejected by a strong outflow. In addition, we show the absolute value of current density in the 


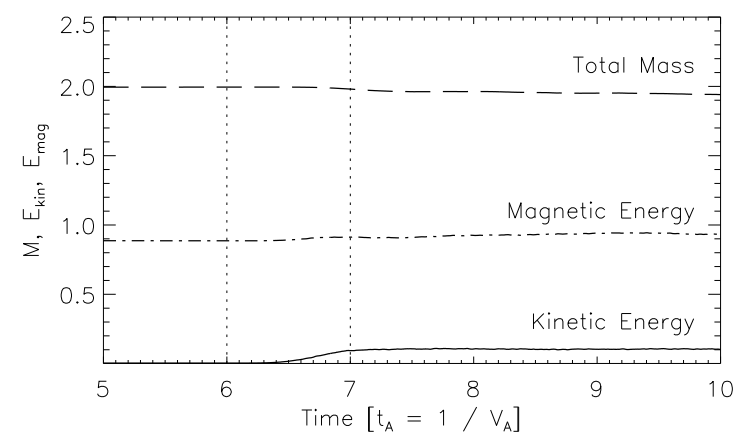

FIG. 8.- Evolution of total mass $M$, and kinetic and magnetic energies, $E_{k i n}$ and $E_{m a g}$, respectively for the stage with turbulence. The kinetic energy $E_{k i n}$ is not amplified in this plot. Two dotted vertical lines bound the period of gradually increasing turbulence. The resistivity in this model is set to $\eta=10^{-3}$ and the shared component of magnetic field $B_{0 z}=0.1$.

right panel of Figure 10 . We see an elongated diffusion region in the middle of the box, where the reconnection process takes place. The maximum of $|\vec{j}|$ does not exceed a value of 25. Evidently our boundary conditions allow for physically realistic inflow and outflow around a central current sheet.

\subsection{Effects of Turbulence}

\subsubsection{Evolution in the Presence of Turbulence}

The essential part of our studies covers the effects of turbulence on reconnection. Our goal was to achieve a stationary state of Sweet-Parker reconnection, described in the previous subsection, and then introduce turbulence at a given injection scale $l_{i n j} \propto k_{i n j}^{-1}$, gradually increasing its strength to the desired amplitude corresponding to a turbulent power $P_{i n j}$. We inject turbulence in a region surrounding the midplane and extending to the distance of around one quarter of the size of the box. This, naturally, limits the injection scale to $k_{f} \lesssim 3$. The transition period, during which we increase the strength of turbulence has a length of one Alfvénic time unit in all models and in the presented model starts at $t=6$. This means that from $t=7$ we inject turbulence with the maximum power $P_{i n j}$.

In Figure 8 we present an example of the evolution of total mass, and kinetic and magnetic energies in a model with $P_{i n}=1.0, k_{f}=8$, and $\eta_{u}=10^{-3}$. We inject turbulence, gradually increasing its strength from $t=6$ to $t=7$. This period is marked by two dotted vertical lines in Figure 8. We see an increase of kinetic energy during this period due to the injection and after $t=7$ all quantities saturate and do not vary much. This means that even in the presence of turbulence and with open boundary conditions our system conserves total mass and energy relatively well.

In Figure9 we show the evolution of reconnection rates $\left\langle V_{i n} / V_{A}\right\rangle$ and more advanced $V_{r e c}$, both described in $\$ 4$ In this plot we recognize an increase of both rates during the introduction of turbulence. After the initial period between $t=6$ and $t=8$, during which the system is adjusting to the new state, we see that both measures coincide and even though they are fluctuating, they reach a stationary state characterized by faster reconnection. A more detailed comparison of the simple and advanced reconnection rate measures is presented in $\$ 5.4$

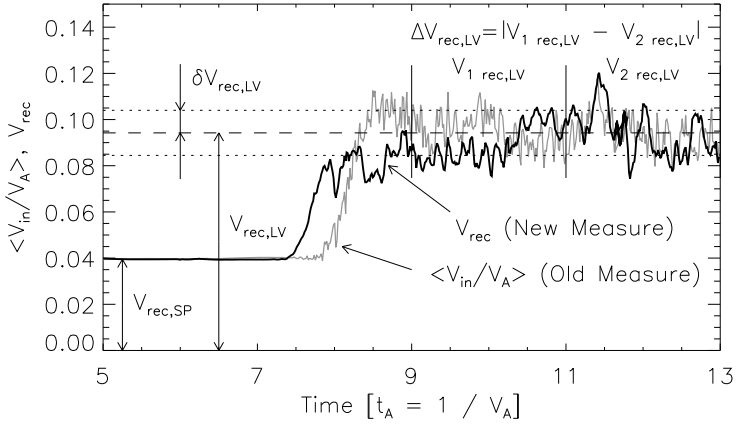

FIG. 9.- Evolution of the reconnection rates, $\left\langle V_{i n} / V_{A}\right\rangle$ (old) and $V_{\text {rec }}$ (new), for the same model as in Fig. 5 In this plot we present the measured rates of the Sweet-Parker reconnection $V_{r e c, S P}$ and during the presence of turbulence, $V_{r e c, L V}$. Symbol $\delta V_{r e c, L V}$ is the time variance. $\Delta V_{r e c, L V}$ is the estimated uncertainty of the measure.

In Figure 9 we also show how we measure the rates of Sweet-Parker reconnection $V_{r e c, S P}$ and LV99 model $V_{r e c, L V}$. Because the reconnection rates fluctuate in the presence of turbulence we also measure their time variance $\delta V_{r e c, L V}$ using standard deviation. In addition to the time variance of $V_{r e c}$, we measure their errors by splitting the averaging region into two subregions and after averaging the rates $V_{1 \text { rec }}$ and $V_{2 r e c}$ over each subregion (see Fig. 9), we take the absolute value of their difference $\Delta V_{r e c}=V_{1 r e c}-V_{2 r e c}$. This difference corresponds to the error of $V_{r e c}$, i.e. it is different from zero if the rate is not constant in time. In all further analysis and presented plots of dependencies we use values estimated in this way.

Before we discuss the main results obtained in these studies, we present and describe the configuration and topology of the velocity and magnetic fields in the presence of turbulence. In Figure 11] we show examples of XY-cuts (upper row) and XZ-cuts (lower row) through the box of the velocity (left panel) and magnetic field (middle panel) topologies with the intensities corresponding respectively to the amplitude of perpendicular components of velocity and magnetic field to a normal vector defining the plotted plane.

The first noticeable difference compared to the SweetParker configuration is a significant change of the velocity and magnetic field topologies. Velocity has a very complex and mixed structure near the midplane, since we constantly inject turbulence in this region (see the left panel in Fig. 11). Although the structure is very complex, most of the velocity fluctuations are perpendicular to the mean magnetic field. This is because we are in the nearly incompressible regime of turbulence (large plasma $\beta$, see Tab. 1) and most of the fluctuations propagate as Alfvén waves along the mean magnetic field. Slow and fast waves, whose strengths are significantly reduced, are allowed to propagate in directions perpendicular to the mean field as well. As a result most of the turbulent kinetic energy leaves the box along the magnetic lines. We observe, however, the efficient bending of magnetic lines at the midplane (see the upper middle plot in Fig. 11). This is not result of a driving, but result of reconnection. In general the interface between positively and negatively directed magnetic lines is much more complex than in the case of Sweet-Parker reconnection. This complexity fa- 

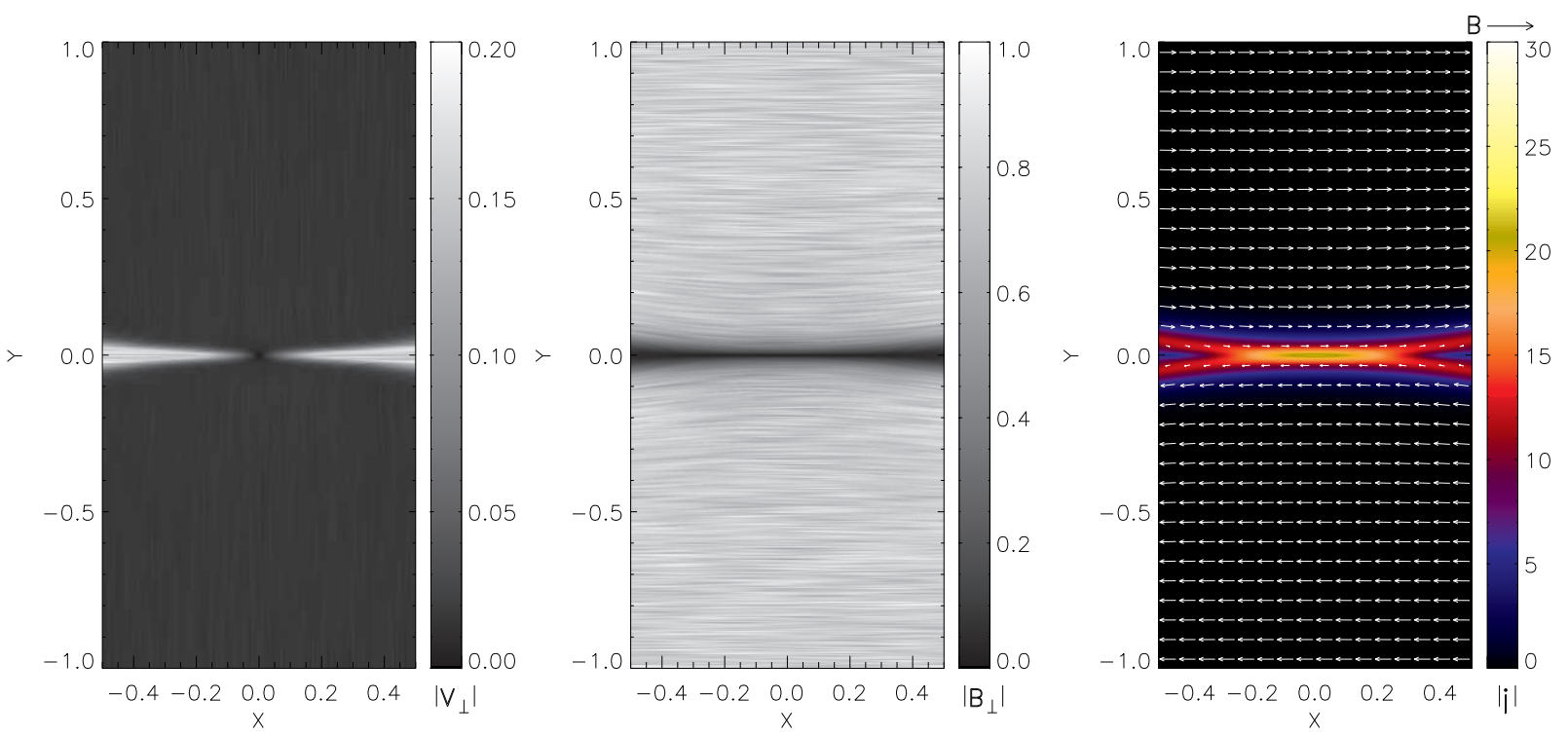

FIG. 10.- Topology and strength of the velocity field (left panel) and magnetic field (middle panel) during the Sweet-Parker reconnection at $t=7$. The strength is calculated from the components of $\boldsymbol{V}$ and $\boldsymbol{B}$ perpendicular to the normal vector of the XY-plane. In the right panel we show the absolute value of current density $|\vec{j}|$ overlapped with the magnetic vectors. The images show the XY-cut through the domain at $Z=0$ at time $t=7$ for a model with $B_{0 z}=0.1, \eta_{u}=10^{-3}, \eta_{a}=0.0$, and the resolution $256 \times 512 \times 256$.
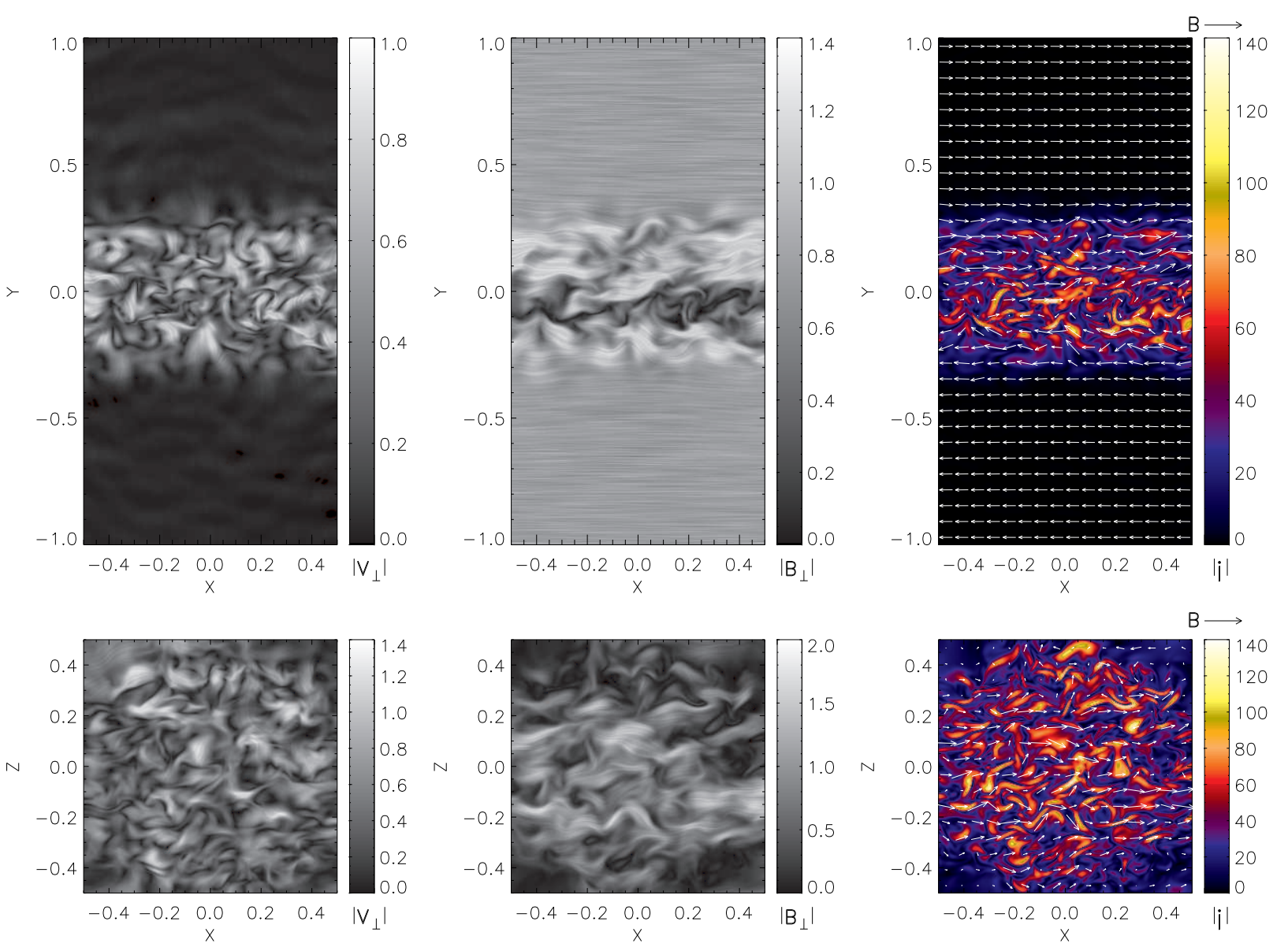

FIG. 11. - Topology and strength of the velocity field (left panel) and magnetic field (middle panel) in the presence of fully developed turbulence at time $t=12$. In the right panel we show distribution of the absolute value of current density $|\vec{J}|$ overlapped with the magnetic vectors. The images show the XY-cut (upper row) and XZ-cut (lower row) of the domain at the midplane of the computational box. Turbulence is injected with power $P_{i n j}=1$ at scale $k_{i n j}=8$. Magnetic field reversals observed are due to magnetic reconnection rather than driving of turbulence, which is subAlfvénic. 
vors creation of enhanced current density regions, where the local reconnection works faster since the current density reaches higher values (see the right panel of Fig. 11). Since we observe multiple reconnection events happening at the same time (compare the right panel of Fig. 11 to the Sweet-Parker case in Fig. 10], the total reconnection rate should be significantly enhanced.

\subsubsection{Dependence on Turbulence Strength}

We ran several models (models PD in Tab. 1) with varying powers of turbulence. All other parameters were kept the same, allowing us to estimate the dependence of the reconnection rate $V_{\text {rec }}$ on the power of injected turbulence $P_{i n j}$.

Figure 12 (left panel) shows the evolution of reconnection speed in models with turbulent power $P_{i n j}$ varying in range by more than one order of magnitude, from 0.1 to 2.0. The evolution of $V_{\text {rec }}$ reaches stationarity in a relatively short period, about one Alfvén time unit, as estimated from the plot. We averaged $V_{\text {rec }}$ from $t=8.2$ to $t=12$. From this plot we see that the reconnection rates are relatively stable. In the right panel of Figure 12 we plot the averaged reconnection speed over the strength of turbulence. Filled symbols represent the averaged reconnection rate in the presence of turbulence. Open symbols represent the reconnection rate during the Sweet-Parker process, i.e. without turbulence. The error bars show the time variance of $V_{r e c}$. The size of symbols indicates the uncertainty in our estimate of the reconnection speed $\Delta V_{\text {rec, } L V}$ normalized to the uncertainty in the reconnection speed during the Sweet-Parker evolution $\Delta V_{r e c, S P}$ using formula size $=2.0-\ln \Delta V_{\text {rec }, L V} / \ln \Delta V_{\text {rec }, S P}$. Meaning that if $\Delta V_{r e c, L V}$ is of order of $\Delta V_{r e c, S P}$ their symbols have the same size.

Fitting a power law to the calculated points indicates that the reconnection speed $V_{\text {rec }}$ scales as $\sim P_{i n j}^{1 / 2}$, as expected from the LV99 prediction (see Eq. 4).

\subsubsection{Dependence on Injection Scale}

We performed similar studies to determine the dependence of the reconnection speed $V_{\text {rec }}$ on the scale at which we inject turbulence, $l_{i n j}$. Keeping the same power of turbulence for all models SD (see Tab. 1) we inject turbulence at several scales, from $k_{i n j}=5$ to $k_{i n j}=25$. At the upper end of this range the turbulence barely broadens the Sweet-Parker current sheet. At the lower end the turbulent eddies are barely contained within the volume within which we excite turbulent motions.

In Figure 13 (left panel) we present the results for this series of models. From the plot we clearly see a strong dependence of the reconnection rate on the injection scale. After the injection of turbulence, models with smaller power injection scales reach smaller values of the reconnection rate $V_{r e c}$. In the model with the largest injection scale $k_{i n j}=5$, reconnection is 5 times faster than the Sweet-Parker rate. After averaging over time, we plot the dependence of $V_{r e c}$ on the injection scale in the right plot of Figure 13

The prediction of LV99 model is that the reconnection rate should scale as $\sim l_{i n j}$. In the case of a strong guide field we see that this relation is observed for small injection scales. However, when $l_{i n j}$ is comparable with length of the volume of driven turbulence the dependence becomes flatter. For a weak guide field the dependence is flatter from the very beginning and is better fit by $V_{r e c} \sim l_{i n j}^{3 / 4}$. This difference requires further investigation.

We see several possible sources for the discrepancy. For instance, the existence of a turbulent inverse cascade can modify the effective $l_{i n j}$. In addition, reconnection can also modify the characteristics of turbulence, such as the power spectrum and anisotropy. We shall study this issue elsewhere, but within this work get satisfied with the qualitative agreement of the predictions and the numerical simulations.

\subsubsection{Dependence on Resistivity}

In the global constraint on the reconnection rate (Eq. 3) derived in LV99, there is no explicit dependence on the resistivity. In order to test this, we performed another set of models (RD in Tab. 1) in which we change the uniform resistivity $\eta_{u}$ only. We expect that in these models we should see at early times the theoretical dependence of the Sweet-Parker reconnection rate $\left(V_{r e c, S P} \sim \eta_{u}^{1 / 2}\right)$, and at late times, after we introduce turbulence, a complete lack of any dependence on resistivity.

In the left panel of Figure 14 we show the evolution of the reconnection rate for a subset of $\mathrm{RD}$ models with $B_{0 z}=0.1$ and with $\eta_{u}$ varying from $5 \cdot 10^{-4}$ to $5 \cdot 10^{-3}$. In this plot we can recognize a strong dependence of the Sweet-Parker reconnection on the uniform resistivity, while in the presence of turbulence the reconnection speed, even though it fluctuates, reaches roughly the same level for all models. This indicates that, as expected, there is little or no dependence of the reconnection rate on resistivity in the presence of turbulence.

In the right panel of Figure 14 we show reconnection rates obtained from the set $\mathrm{RD}$ of models with a weak and strong guide fields, $B_{0 z}=0.1$ and $B_{0 z}=1.0$, respectively. The open symbols show the rates of laminar reconnection $V_{\text {rec,SP }}$. They follow precisely the theoretical relation $V_{r e c, S P} \sim \eta_{u}^{1 / 2}$. Evolving the models further in the presence of turbulence allowed us to test the dependence of the reconnection rates on resistivity in the presence of turbulence. We plot these results using filled symbols. We see that there is virtually no dependence on $\eta_{u}$. Moreover, we see that this property does not depend on the strength of $B_{0 z}$.

These results were obtained in models with a decaying zone of resistivity near the $\mathrm{X}$ boundary. In order to exhibit the importance of properly treating the resistive terms at the outflow boundary conditions we show in Figure 15 values obtained in models with regular open boundary conditions without a zone of decaying resistivity. Here, the open circles show the rates of laminar reconnection with no turbulence. We clearly see a growing departure of these results from the theoretical prediction, $V_{r e c, S P} \sim \eta_{u}^{1 / 2}$ (solid line) for models with large values of uniform resistivity. For $\eta_{u} \gtrsim 2 \cdot 10^{-3}$, laminar reconnection seems to be insensitive to the value of resistivity. This effect is purely numerical and caused by improper handling of boundary conditions when the resistive term in the induction equation starts to dominate. Open diamonds in Figure 15 show the correspond- 

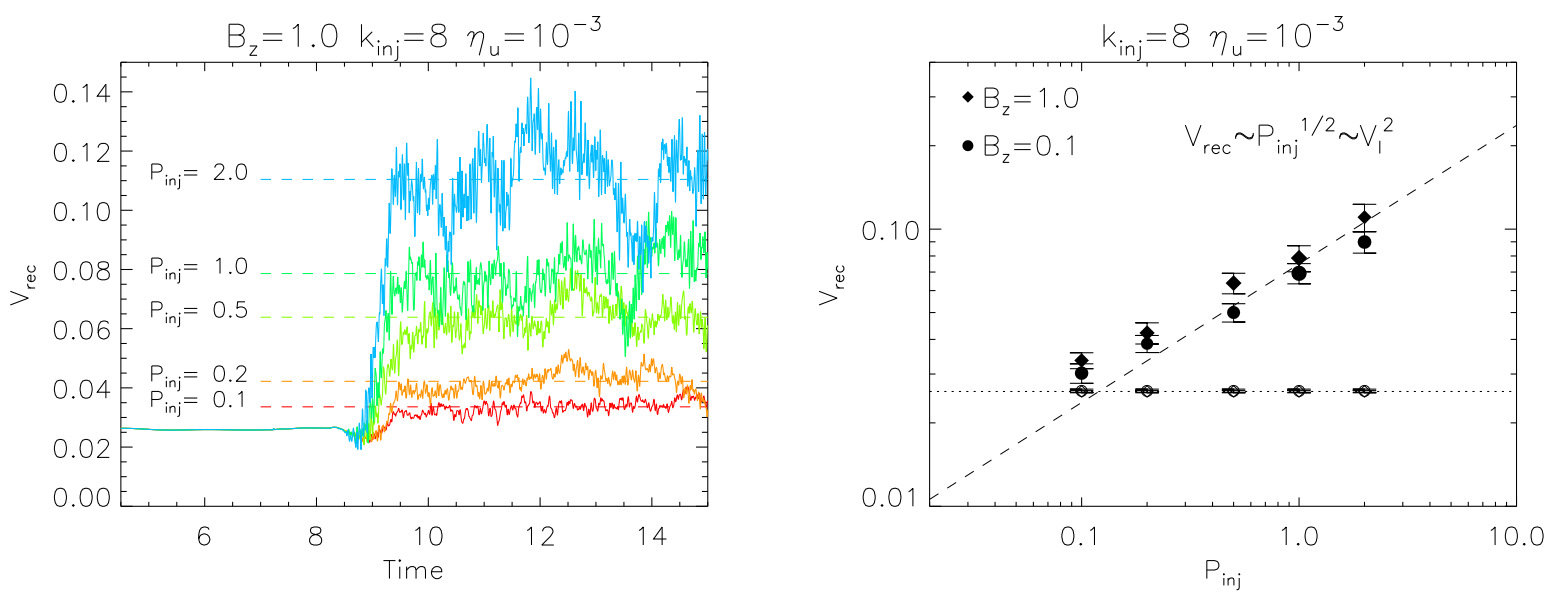

Fig. 12.- Left: Time evolution of the reconnection speed $V_{r e c}$ for models PD (see Table 1) with different powers of turbulence $P_{i n j}$ (see legend). Right: The dependence of the reconnection speed $V_{r e c}$ on $P_{i n j}$. Error bars represent the time variance of $V_{r e c}$. The size of symbols corresponds to the error of $V_{r e c}$ (the way we calculate errors is described in $\$ 5.2$ ).
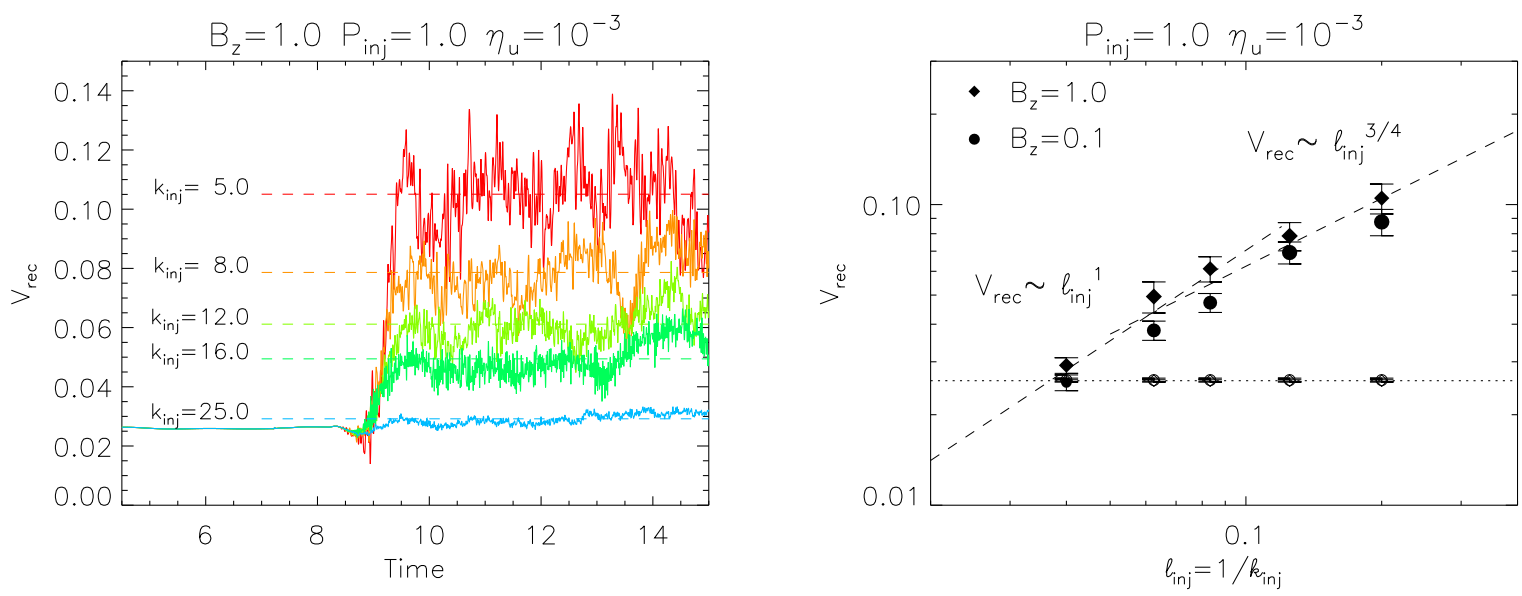

FIG. 13. - Left: Time evolution of the reconnection speed $V_{r e c}$ for models SD (see Table 1) with different injection scale $l_{\text {inj }}$ (see legend). Right: The dependence of the reconnection speed $V_{r e c}$ on $l_{i n j}$. Error bars and the size of symbols have the same meaning as in Fig. 12.
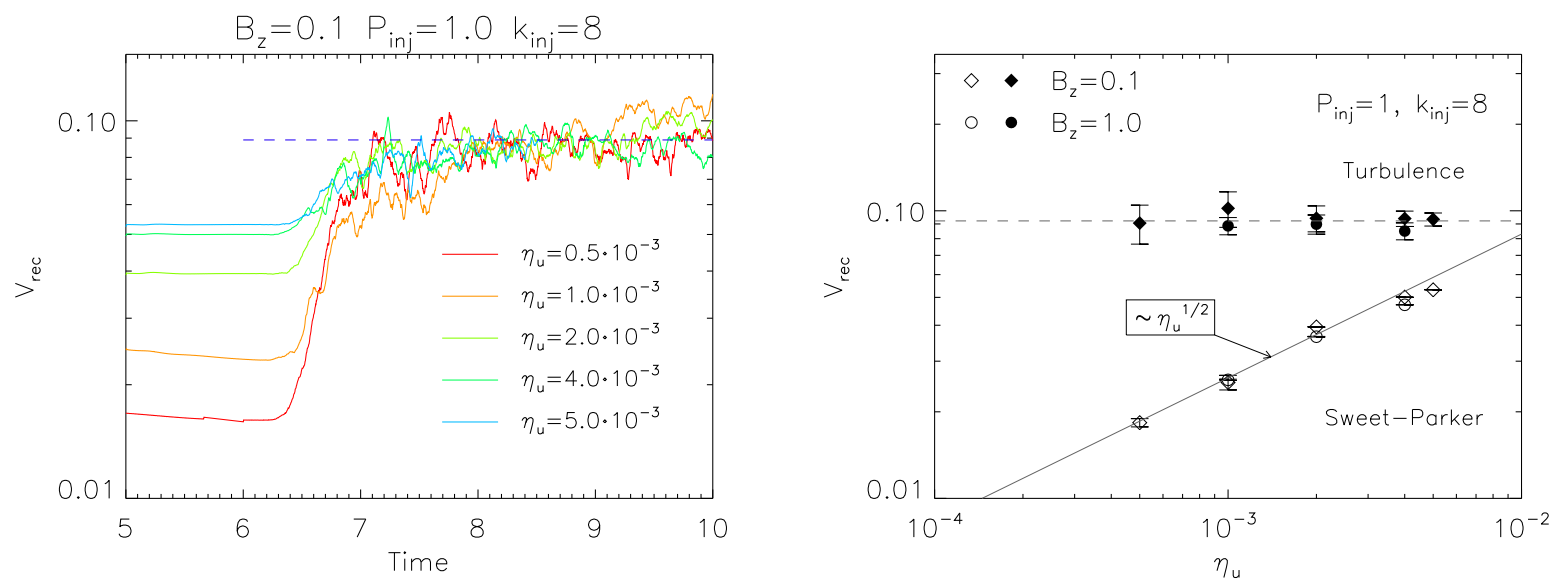

FIG. 14.- Left: Time evolution of the reconnection speed $V_{r e c}$ for models RD (see Table 1) with different resistivity coefficients $\eta_{u}$ (see legend). Right: The dependence of the reconnection rate $V_{\text {rec }}$ on the uniform resistivity $\eta_{u}$. The solid line shows the theoretical dependence in Sweet-Parker model, $V_{\text {rec }, S P} \sim \eta_{u}^{1 / 2}$. Open symbols show Sweet-Parker reconnection rate for different models. Filled symbols show reconnection rates $V_{\text {rec }}$ in the presence of turbulence. Dashed line shows the mean value of reconnection rate averaged over all models during the turbulence stage (all filled symbols). Error bars show the measure of time variance of $V_{r e c}$. 


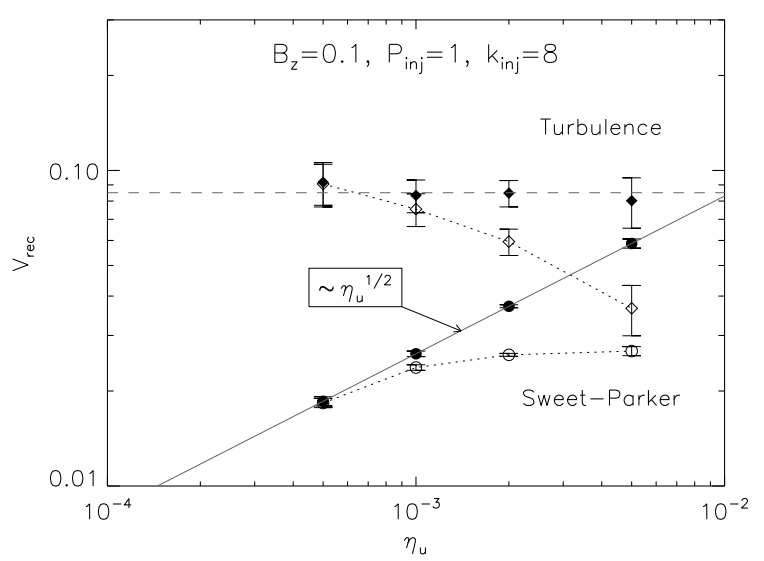

FIG. 15.- Dependence of the reconnection rate $V_{\text {rec }}$ on the uniform resistivity $\eta_{u}$ without the decaying resistivity zone. The solid line shows the theoretical dependence in Sweet-Parker model, $V_{\text {rec,SP }} \sim \eta_{u}^{1 / 2}$. Open circles show Sweet-Parker reconnection rate for different models. Open diamonds show reconnection rates $V_{r e c}$ in the presence of turbulence. Filled symbols show values corrected by the factor calculated from the theoretical dependence (see text).

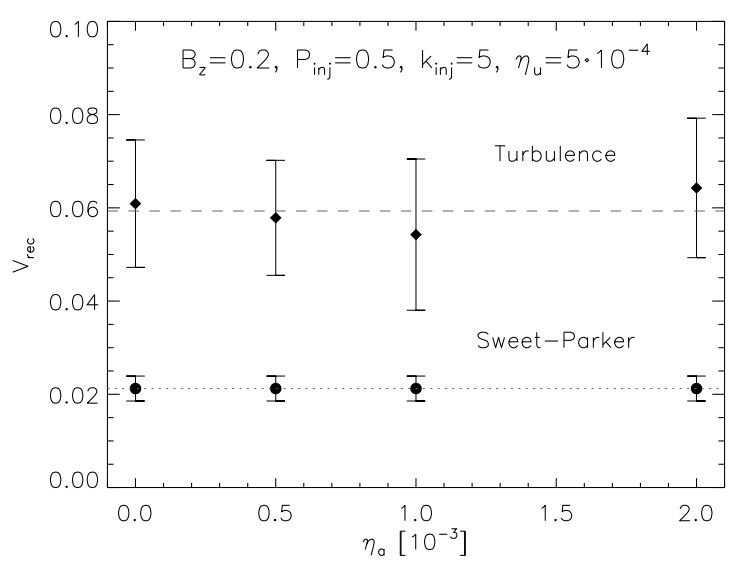

FIG. 16.- Dependence of the reconnection rate $V_{r e c}$ on the anomalous resistivity $\eta_{a}$. Two horizontal lines show the mean reconnection rate averaged over all plotted models during the SweetParker stage and in the presence of turbulence (dotted and dashed lines, respectively). The critical value of the current density $j_{\text {crit }}$ is set to 25.0 in all models plotted here.

ing rates for models with turbulence. Even though initially, for smaller values of resistivity, the reconnection rate seems to be independent of $\eta_{u}$, later on, when the resistivity is larger, it starts to decay with $\eta_{u}$. The problem of boundaries affects the turbulent case as well.

We can test our ability to compensate for the affect of large resistivity on the boundary conditions using the theoretical dependence $V_{\text {rec }, S P} \sim \eta^{1 / 2}$. If we calculate correction factors by taking the ratio of $\eta^{1 / 2} / V_{r e c, S P}$, we can use these coefficients to correct reconnection rate during the turbulent stage. The result is plotted with the filled symbols. The relation signifies virtually no dependence of the $V_{\text {rec }}$ on $\eta$.

In addition to the uniform resistivity dependence, we have studied the dependence on anomalous effects as well. The results of these studies are presented in Figure 16 and show four models with the same uniform re- sistivity $\eta_{u}=5 \cdot 10^{-4}$ and the critical current density $j_{\text {crit }}=25.0$ but with the anomalous resistivity parameter $\eta_{a}$ varying between 0.0 and $2 \cdot 10^{-3}$ (see models $\mathrm{AD}$ in Tab. 1). In Figure 16 we plot the dependence of the reconnection rate on the anomalous resistivity parameter $\eta_{a}$. We see that also the reconnection speed $V_{\text {rec }}$ is insensitive to the value of $\eta_{a}$ to within the variations of the reconnection rate in each model (see the error bars).

In this section we shown that the results of our resistivity studies agree with the LV99 model and indicate no sensitivity to Ohmic resistivity in the presence of turbulence. This is important for two reasons. First, it supports the stochastic reconnection model proposed in LV99. Second, it gives reason to believe that simulations of reconnection and MHD turbulence in astrophysical objects can be successfully applied to real objects, despite the enormous difference between the magnetic Reynolds numbers that can be simulated and the values that actually obtain in stars, accretion disks, and galaxies.

\subsection{Role of the Guide Field}

The robustness of the LV99 model is determined by its application to any configuration of the reconnecting field. Usually textbooks describe the Sweet-Parker model as having opossitely directed field lines, which undergo the reconnection process in the diffusion region and are perfectly antiparallel, since this model is strictly two dimensional. This situation is very particular. In reality, even in the absence of turbulence, magnetic field lines can enter the diffusion region at different angles $\alpha$ (see right panel of Fig. (3), or in other words, an uniform component parallel to the reconnecting field could be present. This component is called a guide field and in our simulations is determined by the value of $B_{0 z}$.

Direct simulations of the LV99 model with a strong guide field of order of the reconnecting component strength encounter problems when the $\mathrm{Z}$ boundaries are open. In our picture, the simulation domain is a box embedded in a large scale configuration of magnetic field around the interface of two volumes characterized by the different direction of magnetic lines. In this picture, magnetic lines crossing our domain extend to infinity. This means that any force acting on a field line in the domain will feel the tension of that line, so the general configuration of the field line will not change. It only encounters a small local perturbation. When the open boundary conditions are applied, the force acting on a magnetic line is not bounded by the magnetic tension anymore, and in the presence of shear flows generated by turbulence, magnetic line ends attached to the boundaries can slip along them changing the global topology of magnetic field. Moreover, the change of direction of the field transforms $B_{z}$ into $B_{x}$ component, which suppresses the inflow of fresh unreconnected flux. This is an artificial and undesirable situation.

There are two solutions to this problem. First, we can construct a special type of open boundary conditions, which fixes magnetic lines at some distance far from the box. Then, at the boundary, we would have to extrapolate the magnetic field lines and calculate the required tension from this condition. This approach, however, seems to be complex and requires additional conditions assumed a priori at the boundaries. Thus, we have chosen another approach by applying periodic boundary 

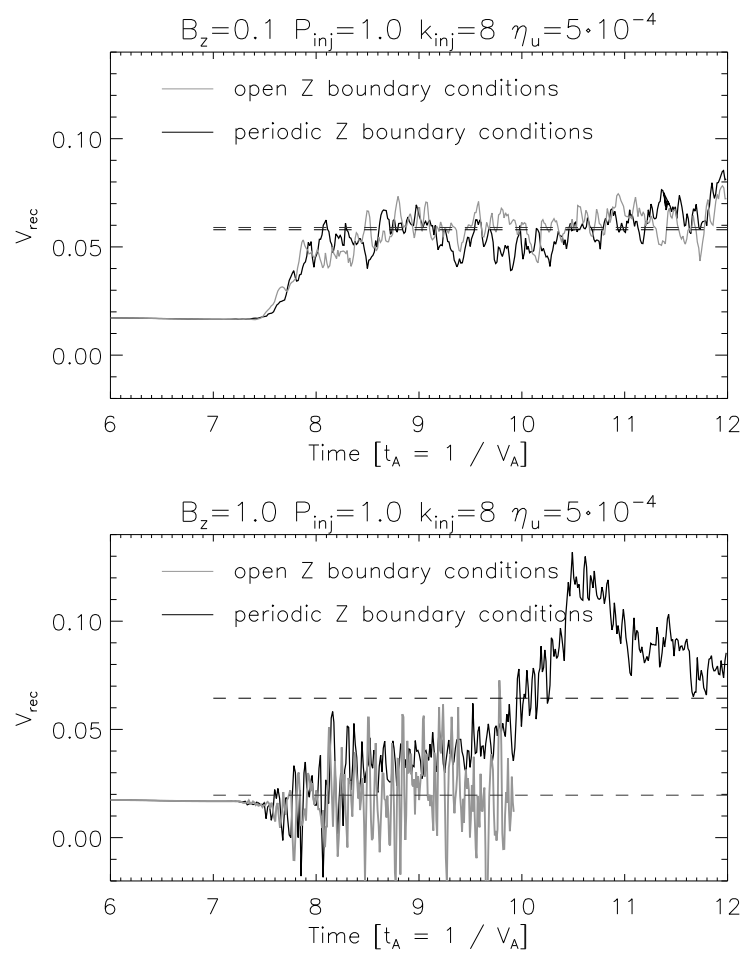

FIG. 17.- Demonstration of the importance of boundary condition along Z-direction. Upper: Comparison of two models with the same conditions but different types of $\mathrm{Z}$ boundaries for a weak $B_{0 z}$. Lower: Comparison of two models with the same conditions but different types of $\mathrm{Z}$ boundaries for a strong $B_{0 z}$.

conditions along the $\mathrm{Z}$ direction. This is much simpler and does not limit our model, i.e. it does not influence the inflow and outflow of the magnetic flux.

In Figure 17we show the comparison of $V_{\text {rec }}$ for models with a weak (top) and strong (bottom) guide fields $B_{0 z}$. Each plot contains two otherwise identical models, but one with open boundaries along $\mathrm{Z}$ direction and another with periodic boundaries. As we see, in the case of weak $B_{0 z}$ there is no difference if we apply open or periodic boundaries. In both models, mean values of reconnection speed during the presence of turbulence are almost identical (see black and grey dashed lines in top panel of Fig. (17). However, once we start increasing the strength of the guide field, our choice of the boundary conditions starts to be important. In the case of open boundaries along the $\mathrm{Z}$ direction, the mean reconnection rate is suppressed by the strong turbulent shear of $B_{z}$, which is clearly seen in the bottom plot of Figure 17. Applying periodic boundaries along the $\mathrm{Z}$ direction we can restore the fast reconnection rate, but the system needs more time to adjust to a steady state. This is caused by the fact that after starting injection of turbulence, part of the injected kinetic energy is converted into magnetic energy, thus the relaxation of the system takes place more slowly than in the case of weak $B_{z}$. This demonstration shows that the choice of applied boundary conditions along the $\mathrm{Z}$ direction is important and has a crucial influence on the reconnection rate, at least in the case of strong magnetic guide field.

In Figure 18 we show four models (set BD in Tab. 1) with different strengths of the guide field $B_{0 z}$. This plot shows that the reconnection speed is comparable over a broad range of $B_{0 z}$ (and the angle $\alpha$ ), which means

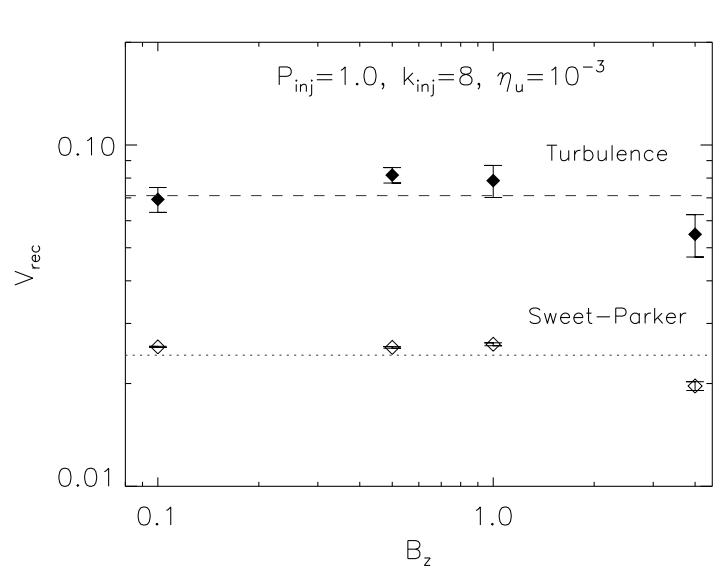

FIG. 18.- Dependence of the reconnection rate $V_{\text {rec }}$ on the strength of the guide field $B_{0 z}$.

that reconnection will work with similar efficiency for any configuration of magnetic field in which the lines of the magnetic field enter the diffusion region at different angles. The studied models with $0.1 \leq B_{0 z} \leq 1.0$ cover range of $\alpha$ from $0^{\circ}$ to $90^{\circ}$. For a given power input a larger guide field is expected to correspond to a smaller amount of field wandering and therefore a smaller reconnection speed (see the model with $B_{0 z}=4.0$ corresponding to $\alpha \approx 152^{\circ}$. We, however, do not see a prominent dependence on the shared component of magnetic field, which calls for further studies of the effects of the strong guide field.

\subsection{Comparison of the Old and New Measures}

In Figure 19 we show a comparison of the reconnection rate obtained using the old and new measures as a function of the power of turbulence (left plot) and the injection scale (right plot). In the case of the turbulent power dependence we used two different sets of models for each method. For the old method of $V_{\text {rec }}$ estimation the guide field $B_{z}=0.2$, and the uniform resistivity $\eta=5 \cdot 10^{-4}$. The guide field in new models is set to 0.1. The injection scales are $k_{i n j}=5$ and 8 , for the old and new models, respectively. Dashed line shows the LV99 dependence $V_{\text {rec }} \sim V_{T}^{2}$.

The old measure models were calculated with a more dissipative scheme based on the HLL solver instead of HLLD, with a higher value of numerical resistivity. During the Sweet-Parker stage we note some difference in the values of reconnection rate between two sets of models resulting from the different values of uniform resistivity $\eta_{u}$ in the models and other parameters, and different type of schemes used to evolve the numerical models. After the injection of turbulence, as we shown in Figures 14 and 16, during the stage of the presence of turbulence, the reconnection rate is insensitive to the value of resistivity. In Figure 19 we see that both relations, using the old and new methods, show the same dependence, even though they were fitted to two different sets of models. This means that the reconnection rate dependence on the injected power is not sensitive to the strength of the guide field $B_{z}$, the injection scale or the value of uniform resistivity and the numerical diffusivity of the method used in solving the MHD equations.

In the right plot of Figure 19 we see a comparison of the 

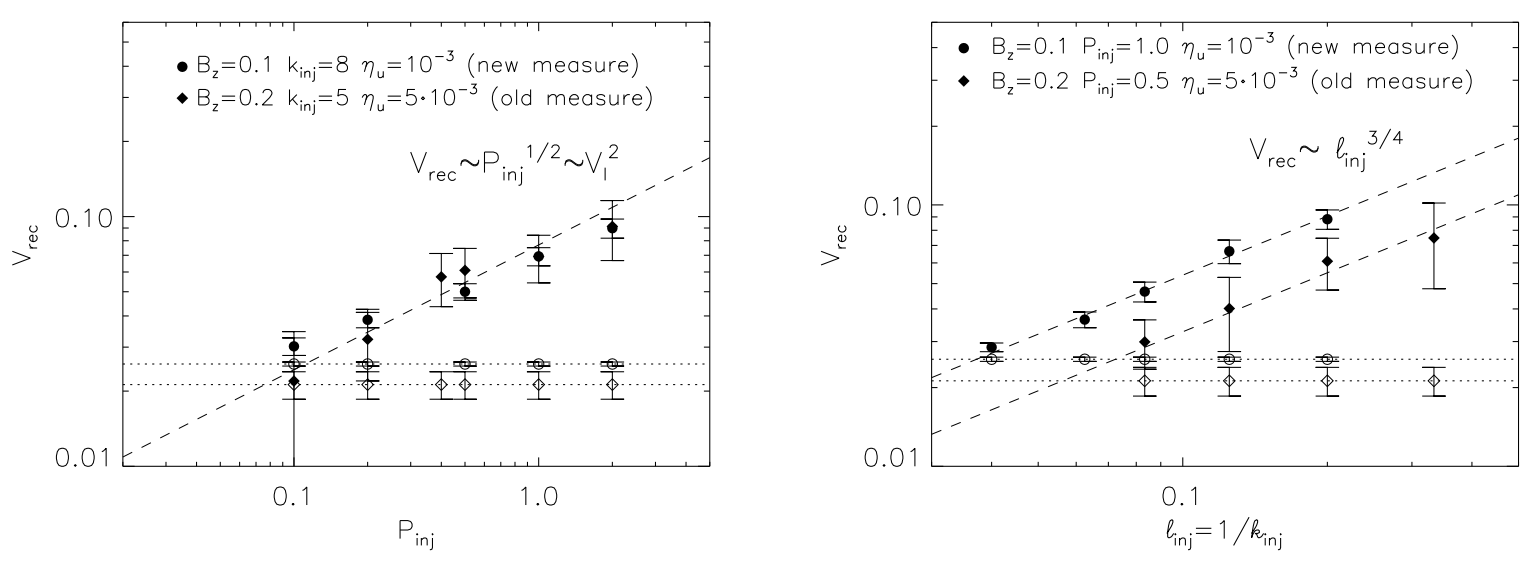

Fig. 19.- Comparison of the old and new reconnection rate estimates. In the left plot we show the dependence of the reconnection rate $V_{r e c}$ on the power of turbulence $P_{i n j}$ (diamonds) using both methods. The corresponding Sweet-Parker rates, without the presence of turbulence, are shown using x-symbols. In the right plot we show the dependence of the reconnection rate $V_{r e c}$ on the injection scale $l_{i n j}$ (diamonds). Again, the Sweet-Parker rates are shown using x-symbols.

old and new measures of $V_{\text {rec }}$ as a function of the injection scale $l_{i n j}$. Similar to the dependence on the power of turbulence, this dependence does not change and in both cases is $V_{r e c} \sim l_{i n j}^{3 / 4}$. This confirms again that the dependence of the reconnection rate $V_{\text {rec }}$ on the properties of the turbulence do not change its character. The only difference seen in both relations is the amplitude of the reconnection rate. This difference comes from the fact, that in the old measure models we used weaker turbulence $\left(P_{i n j}=0.5\right.$ vs. $\left.P_{i n j}=1.0\right)$. Another difference could result from the fact that we include more terms in the elaboration of the new reconnection rate, such as the boundary shear, the boundary resistive terms, the time derivative of the absolute value of $\left|B_{x}\right|$. These terms can have non-zero time averages, although we expect them to have at least stationary values.

The results showed here confirms that the new and old methods of estimating the reconnection rate reveal the same dependence on the power of turbulence and injection scale. It signifies that the old measure $\left\langle V_{i n} / V_{A}\right\rangle$ and new one $V_{r e c}$ are both adequate in determining the speed of reconnection and more importantly, that they measure precisely the reconnection rate in our system excluding all other processes, which could contribute to the plasma flow, but not to the reconnection process itself. It is also important that these results are independent of the numerical scheme or the initial conditions.

\subsection{Non-Reconnection Case}

The results presented so far indicate an agreement with the LV99 model. We see that the presence of turbulence near current sheets enhances reconnection significantly. However, since our testing is limited to numerical models, which have explicitly defined boundaries, our results may be sensitive to interactions between turbulence and the boundary conditions implemented in the numerical model. These interactions could stimulate a flow through the box resulting in a false, non-zero reconnection rate without the presence of reconnection itself. Moreover, the measures of reconnection rate that we applied here may indicate the existence of other processes contributing to the plasma flow and related to the turbulence itself rather than reconnection. In order to test the reliability

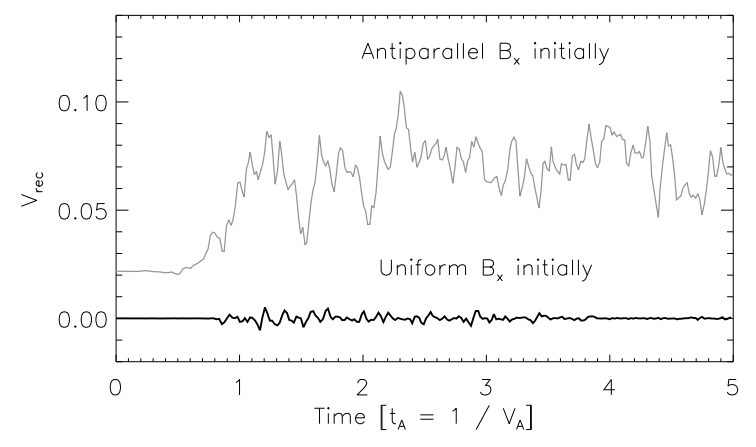

FIG. 20.- Comparison of the reconnection rate $V_{\text {rec }}$ for two models with the same set of parameters, $P_{i n j}=1.0, k_{f}=8, B_{0 z}=$ $0.1, \eta_{u}=10^{-3}$, but different initial configurations of magnetic field: reversed $B_{x}$ (grey) and uniform $B_{x}$ (black). Both models were run with the same resolution $128 \times 256 \times 128$

of our numerical model and the methods of reconnection rate estimation we present below a comparison of two models with the same set of parameters, but with different initial configurations of magnetic field, namely, one with the antiparallel $B_{x}$ as used in all numerical models presented here and another with the uniform $B_{x}$ where is no laminar reconnection before we start injecting turbulence. From the latter model one would obviously expect the lack of reconnection and zero reconnection rate $V_{\text {rec }}$.

In Figure 20 we show the evolution of reconnection rate $V_{r e c}$ for two models with the same set of parameters, $P_{i n j}=1.0, k_{i n j}=8, B_{0 z}=0.1$, and $\eta_{u}=10^{-3}$. Models were started from different initial configurations of magnetic field. The initial configuration of $\boldsymbol{B}$ in the first model is taken from the steady-state laminar reconnection of Sweet-Parker type. In the second model we set uniform magnetic field $B_{x}=1.0$ initially. Starting the evolution from these conditions we inject turbulence in the same way in both models, increasing its strength gradually through the period of one Alfvénic time unit. Then, turbulence is injected with the power of constant value of $P_{\text {int }}=1.0$.

Figure 20 shows a clear difference between the reconnection rates in both models, i.e., in the case of initial Sweet-Parker configuration further evolution of $V_{r e c}$ is similar to the results presented in the previous sections. 
The reconnection rate grows from a value corresponding to the Sweet-Parker rate of about 0.02 to higher values when it saturates at time $t \approx 1.4$ at the level of $V_{\text {rec }} \approx 0.07$. Comparing this to the evolution of $V_{\text {rec }}$ in the model with the initial uniform field we see only small fluctuations of reconnection rate around zero, what indicates the absence of global reconnection in the system.

Results presented in this subsection indicate essentially a lack of reconnection in the case of uniform initial field, what means that the enhancement of reconnection rate in the LV99 model is resulting from an influence of turbulence on the laminar reconnection process and not a result of turbulence itself.

\section{DISCUSSION}

\subsection{Relation to Other Studies of Turbulent Reconnection}

Our simulations presented in this paper test the LV99 model of reconnection. At the same time, the notion that magnetic field stochasticity might affect current sheet structures is not unprecedented. For instance, some papers have concentrated on the effects that turbulence induces on the microphysical level. In particular, Speiser (1970) showed that in collisionless plasmas the electron collision time should be replaced with the electron retention time in the current sheet. Also Jacobson \& Moses (1984) proposed that the current diffusivity should be modified to include the diffusion of electrons across the mean field due to small scale stochasticity. Our simulations are performed at the MHD level and do not take these effects into account ${ }^{8}$.

$$
\text { "Hyper-resistivity" }
$$

Bhattachariee \& Hameiri

(Strauss

1986;

Hameiri \& Bhattacharjee 1987; Diamond \& Malkov 2003 ) is a more subtle attempt to derive fast reconnection from turbulence within the context of mean-field resistive MHD.The form of the parallel electric field can be derived from magnetic helicity conservation. Integrating by parts one obtains a term which looks like an effective resistivity proportional to the magnetic helicity current. There are several assumptions implicit in this derivation. The most important objection to this approach is that by adopting a mean-field approximation, one is already assuming some sort of small-scale smearing effect, equivalent to fast reconnection. Furthermore, the integration by parts involves assuming a large scale magnetic helicity flux through the boundaries of the exact form required to drive fast reconnection. As we are not aware of any attempts to test the idea of "hyper-resistivity" numerically, we shall not discuss this concept further.

Strauss (1988) examined the enhancement of reconnection through the effect of tearing mode instabilities within current sheets. However, the resulting reconnection speed enhancement is roughly what one would expect based simply on the broadening of the current sheets due to internal mixing. Shibata \& Tanuma (2001) extended the concept suggesting that tearing may result

\footnotetext{
8 These effects will usually be small compared to effect of a broad outflow zone containing both plasma and ejected shared magnetic flux. Moreover, while both of these effects will affect reconnection rates, they are not sufficient to produce reconnection speeds comparable to the Alfvén speed in most astrophysical environments.
}

in fractal reconnection taking place on very small scales. Waelbroeck (1989) considered not the tearing mode, but the resistive kink mode to accelerate reconnection.

We do not expect either tearing or kink modes to allow us to evade the constraints on the global plasma flow that leads to slow reconnection speeds ${ }^{9}$. The effects of tearing modes may be important for the initiation of turbulence in the situation when the initial configuration is laminar (see Dahlburg et al. 1992; Dahlburg \& Karpen 1994; Dahlburg 1997; Ferraro \& Rogers 2004; Lazarian \& Vishniac 2008). We plan to study this effect elsewhere. However, once turbulence is initiated reconnection should proceed independently of the tearing mode. Straus' idea is closely related to recent attempts to explain the existence of the observed thick reconnection region in the context of numerical models of collisionless reconnection (Ciaravella \& Ravmond 2008) and predictions of particle acceleration within these regions (Drake et al. 2006a). Here we do not appeal to collisionless effects, but demonstrate both thick reconnection regions and fast reconnection in the presence of turbulence.

$$
\text { The closest study to ours was done }
$$
by Matthaeus \& Lamkin (1985) (see also Matthaeus \& Lamkin 1986). The authors studied $2 \mathrm{D}$ magnetic reconnection in the presence of external turbulence. An enhancement of the reconnection rate was reported, but the numerical setup precluded the calculation of a long term average reconnection rate. A more recent study along the approach in Matthaeus \& Lamkin (1985) is one in Watson et al. (2007), where the effects of small scale turbulence on 2D reconnection were studied and no significant effects of turbulence on reconnection were reported for the setup chosen by the authors. The major differences from the present study stem from the fact that we test a 3D model of reconnection, as the LV99 depends on effects, e.g. field wandering, that happen only in $3 \mathrm{D}$. Thus within the present study we do not address the question of what is happening to $2 \mathrm{D}$ reconnection in the presence of turbulence, appealing instead to the 3D nature of astrophysical reality (see more in 6.4 ).

Finally, we would like to stress that our calculations do not attempt to validate the concept of turbulent diffusivity frequently employed in the mean field dynamo (see Moffat 1978; Blackman \& Field 2008). This concept appeals to the alleged ability of hydrodynamic motions to mix magnetic lines on very small scales, as if the magnetic field were a passive scalar. Naturally, this assumption is wrong for any astrophysical field of dynamical importance. In our calculations, on the contrary, the magnetic field energy exceeds the kinetic energy and thus, the turbulence is weak. Instead of mixing magnetic lines, as in the case of "magnetic turbulent diffusivity" creating reversals of magnetic fields on the smallest scales, our

\footnotetext{
${ }^{9}$ Sometimes the growth rate of the resistive modes is erroneously identified with the reconnection speeds. This does not account for the global outflow of the matter constraint that should be satisfied for reconnection of magnetic field. When this is done, the results are different. For instance, LV99 equated the shearing rates arising from the global outflow and the rate of the tearing mode growth at the scale of the reconnection region. They obtained the reconnection speed $V_{A} R_{m}^{3 / 10}$, which was faster than the Sweet-Parker rate, but incredibly slow for most astrophysical systems.
} 
driving is only able to induce wandering of the magnetic field lines within a well-defined mean direction of the magnetic field.

In general, it is important not to confuse stochastic reconnection with the ill motivated concept of turbulent diffusivity. For instance, Kim \& Diamond (2001) addressed the problem of stochastic reconnection by calculating the turbulent diffusion rate for magnetic flux inside a current sheet. They obtained similar turbulent diffusion rates for both two dimensional and reduced three dimensional MHD (2D with the Z-components of velocity and magnetic field). In both cases the presence of turbulence had a negligible effect on the flux transport. The authors pointed out that this would prevent the anomalous transport of magnetic flux within the current sheet and concluded that both $2 \mathrm{D}$ and $3 \mathrm{D}$ stochastic reconnection proceed at the Sweet-Parker rate, even if the individual small scale reconnection events happen quickly, which apparently contradicts our calculations in the paper.

We would like to stress that the turbulent diffusion rates within the current sheet are irrelevant to the process of stochastic reconnection (see discussion in LVC04). The basic claim in LV99 is that the realistic magnetic field topologies allow multiple connections between the current sheet and the exterior environment, which would persist even if the stochastic magnetic field lines are stationary ("frozen in time") before reconnection happens. This leads to global outflow constraints which are weak and do not depend on the properties of the current sheet. In particular, the analysis in LV99 assumed that the current sheet thickness is determined purely by Ohmic dissipation and that turbulent diffusion of the magnetic field is negligible inside and outside of the current sheet.

\section{2. $2 D$ versus $3 D$ reconnection}

The fact that our study is in $3 \mathrm{D}$ is essential, as the LV99 model is intrinsically three dimensional. The general picture is of tangled field lines, with reconnection taking place via a series of "Y-points" or modified SweetParker sheets distributed in some fractal way throughout the turbulence. A large scale Sweet-Parker sheet will be replaced by a more fractured surface, but the current sheets will occupy a vanishingly small fraction of the total volume and the field reversal will remain relatively well localized. The model predicts that the reconnection speed would be approximately equal to the strong turbulent velocity with a modest dependence on the ratio of the eddy length to the current sheet length. There should be no dependence on resistivity. The major results contained in our figures showing the dependence of the reconnection speed on resistivity, input power and input scale agree with the quantitative predictions of the LV99 model. We are not aware of any competing models to compare our simulations with.

In the absence of quantitative model to be tested, simulations aimed at studying reconnection speed have been done in 2D. This allowed to achieve higher resolutions (compared to that contemporary available in 3D) but substantially constrained magnetic field dynamics. For instance, the 2D study in Matthaeus \& Lamkin (1986) stresses the importance of turbulence for modifying the character of magnetic reconnection and specifies heating and transport as the effect of particular significance, as well as formation of Petscheck-type "X-points" in 2D turbulence.

As we mentioned earlier, LV99 appeals to "Y-points" and heating does play a role in it. Our simulations are also isothermal. Kim \& Diamond (2001) showed that the transport of magnetic flux is not enhanced to the reconnection zone. At the same time, field wandering is the essential feature of $3 \mathrm{D}$ reconnection, which is absent in the $2 \mathrm{D}$ case. In our forthcoming paper (Kulpa-Dybel et al. 2009) we shall demonstrate the difference of $2 \mathrm{D}$ and $3 \mathrm{D}$ reconnection using direct numerical simulations ${ }^{10}$.

\subsection{Reconnection in collisionless and collisional plasma}

The LV99 model of reconnection is applicable to the collisional medium. For instance, it is applicable to the interstellar medium (known to be both turbulent and magnetized), for which one cannot apply the Hall-MHD reconnection (Yamada 2007). This is a relieving news for the interstellar medium, star formation and Solar simulations (Ostriker et al. 2001; McKee \& Ostriker 2007), as numerical reconnection in MHD codes is fast. Note, that the requirement of being collisionless in terms of magnetic reconnection is different from the usual use of the term in astrophysics. For instance, for the diffuse ISM the collisionallity parameter is $\omega_{c} \tau_{e}$, where $\omega_{c}$ is cyclotron frequency of electrons and $\tau_{e}$ is the collisional time for an electron. However, for Hall-MHD reconnection to be applicable the criterion is different. It is required that the Sweet-Parker current sheet $\delta_{S P}$ width be less than the ion inertial length $d_{i}$. Thus the "reconnection criterion for media to be collisionless" is $\left(L / d_{i}\right)^{1 / 2} /\left(\omega_{c} \tau_{e}\right)<1$, which presents a much severe constraint on the possible rate of collisions. As a result, magnetic reconnection happens mediated by the Hall-MHD only if the extend of the contact region $L$ (see Fig. 1) does not exceed $10^{12} \mathrm{~cm}$. Magnetic fields in the ISM should interact over much larger scales.

Astrophysical environments also contain media to which the Hall-MHD reconnection is applicable, e.g. Solar corona, interplanetary medium. Is LV99 model applicable to such environments? The answer is positive if the level of turbulence is high enough. Indeed, the reconnection on microscales can happen fast, i.e. in the Hall-MHD fashion. However, this may not change the global reconnection rate. Indeed, the LV99 model shows that even with relatively slow Sweet-Parker reconnection at microscales the global reconnection is limited not by Ohmic resistivity, but the rate of magnetic field wondering. In fact, anomalous resistivity that we use in some of our numerical runs is a proxy for the Hall-MHD effect on reconnection and we do not see any dependence of the reconnection rate on the anomalous resistivity (see Fig. 16). We believe that the Hall-MHD local reconnection of magnetic fields is taking place interplanetary medium, which is being tested by local in-situ measurements, while the global reconnection rates are determined by magnetic field wandering as prescribed in LV99. Future dedicated numerical experiments and space measurements should test this idea.

\subsection{Reconnection in Partially Ionized Gas}

10 To our best knowledge no quantitative predictions are currently available for the $2 \mathrm{D}$ reconnection. 
The LV99 model of reconnection deals with either fully ionized plasma or with plasmas where the neutrals constitute less than $10 \%$ of species. For higher percentage of the neutrals, the MHD-type turbulent cascade is truncated by the viscosity of neutrals. How does reconnection happen in a partially ionized gas?

A partially ionized plasma fills a substantial volume within our galaxy and the earlier stages of star formation take place in a largely neutral medium. This motivates our study of the effect of neutrals on reconnection. The role of ion-neutral collisions is not trivial. On one hand, they may truncate the turbulent cascade, reducing the small scale stochasticity and decreasing the reconnection speed. On the other hand, the ability of neutrals to diffuse perpendicular to the magnetic lines allows for a broader particle outflow and enhances reconnection rates.

Reconnection in partially ionized gases before the introduction of the LV99 model looked hopelessly slow. For instance, in Vishniac \& Lazarian (1999, henceforth VL99) we studied the diffusion of neutrals far from the reconnection zone assuming the anti-parallel magnetic lines. The ambipolar reconnection rates obtained in VL99, although large compared to the Sweet-Parker model, are insufficient either for fast dynamo models or for the ejection of magnetic flux prior to star formation. In fact, the increase in the reconnection speed stemmed entirely from the compression of ions in the current sheet, with the consequent enhancement of both recombination and ohmic dissipation. This effect is small unless the reconnecting magnetic field lines are almost exactly antiparallel (VL99, see also Heitsch \& Zweibel 2003a,b). Any dynamically significant shared field component will prevent noticeable plasma compression in the current sheet, and lead to speeds practically indistinguishable from the standard Sweet-Parker result. Since generic reconnection regions will have a shared field component of the same order as the reversing component, the implication is that reconnection and ambipolar diffusion do not change reconnection speeds significantly.

LCV04 presented a model of turbulence in a partially ionized gas. This model agrees well with numerical simulations available as the limiting case which can be characterized by one fluid with a high Prandtl number (see Cho \& Lazarian 2002, 2003). Using this model LVC04 described field wandering, which is the core of the LV99 model of reconnection. They showed that the magnetic reconnection proceeds fast, both in the diffuse interstellar and molecular cloud partially ionized gas.

In the paper above we employed one-fluid code and therefore we could not test the predictions in LVC04. However, LVC04 model at its core is based on the ideas presented in LV99. Therefore, our successful testing of LV99 model provides us with optimism in relation to the LVC04 model. The latter should be tested with a two fluid code capitalizing on our present experience with the boundary conditions, turbulent driving and the ways of measuring of reconnection rate obtained in this paper. Needless to say, the numerical confirmation of fast reconnection in partially ionized gas would open wide prospects for quantifying processes taking place in molecular clouds, including the enigmatic processes of star formation. For instance, Shu et al. (2006) showed that magnetic field is removed from star-forming cores faster than allowed by the standard ambipolar diffusion scenario (see Tassis \& Mouschovias 2005a,b). Shu et al. (2007) proposed a mechanism that required efficient reconnection of magnetic loops based on the "hyper-resistivity" concept. As we discussed earlier, this concept is not selfconsistent and is therefore problematic. Fast magnetic reconnection in the partially ionized gas could serve a similar purpose.

\subsection{The Current Simulations and Remaining Problems}

In this paper we have performed extensive numerical testing of the LV99 model of magnetic reconnection. A advantage of this model is that it is very generic and does not appeal to any particular plasma effects and/or initial boundary conditions. In many respects, it is a natural generalization of the Sweet-Parker model to the case when turbulence is present. It does not require the current sheet to open up, as is required in the Petscheck reconnection model. The only requirement is the presence of field line wandering, which is a well documented aspect of magnetic turbulence (see LVC04 and ref. therein).

Our results indicate that the reconnection speed increases in the presence of weak turbulence in a manner consistent with the LV99 predictions. In particular, the reconnection speed $V_{\text {rec }}$ (Eq. 30) shows a strong dependence on the characteristics of the turbulence, such as the rate of energy dissipation and the scale of injection. It is very important that we observe no explicit dependence on the fluid resistivity, which is the requirement for fast magnetic reconnection in astrophysical environments.

To make progress in simulating the weakly stochastic reconnection, we had to develop a new numerical set up, which included both driving turbulence over a part of the box, inflow conditions for the incoming flux and outflow conditions for the reconnected flux. This enabled us to study both the transitional regimes of reconnection and the steady state one. In comparison, simulations in the periodic box would not be instructive, as stochastic reconnection is dynamic and does not reach a stationary state in one crossing time.

In addition, we have developed a new way of measuring the reconnected flux (see $\S$ 4). This was required as worries were associated with the accuracy of the "intuitive measure", which is the rate with which the unreconnected flux enters the computational box at the inflow boundaries. Potentially, the "intuitive measure" could overestimate the reconnected flux if the unreconnected magnetic lines escape through the leaky outflow boundaries of the computation box. The new measure accounts for such a loss of magnetic flux.

To our satisfaction, we found that the new and old measures provide consistent results both in the case of no turbulence and with turbulence, which means that the effect of the outflow of unreconnected field was subdominant for the situations when the magnetic fluxes were intersecting at an appreciable angle. For the limiting case, however, when the large scale reconnection was absent, i.e. when the corresponding angle between the two fluxes was zero and without turbulence magnetic fields were all parallel, the "intuitive measure" was a non-zero value, while the new measure of reconnection gave zero reconnection rate. This shows the new measure is more reliable. 
Our results confirm that the reconnection of magnetic field in the presence of turbulence is independent of Ohmic resistivity, allowing for fast reconnection. In addition, we showed that the reconnection rate does not depend on the anomalous resistivity, which proves that the LV99 model of reconnection is also applicable to collisionless plasmas and may dominate reconnection in such systems if the level of turbulence is efficiently high. Note, that according to Lazarian \& Vishniac (2008) turbulence in a system may be successfully generated by the release of magnetic energy through reconnection.Thus, we anticipate that the initial reconnection in a collisionless plasma may be induced through the Hall reconnection, but later the resulting MHD turbulence will determine the reconnection rate.

Apart from the prediction in LV99 that the reconnection rate should be independent of resistivity, we have also tested the quantitative scalings of the reconnection rates presented in LV99. In particular, we tested the dependence of the reconnection rate on the turbulent injection power. Our numerical results confirm the LV99 prediction $V_{\text {rec }} \sim V_{l}^{2}$ which, as we explained earlier, translates into $V_{r e c} \sim P_{i n j}^{1 / 2}$ (see Eq. (4)).

We do not expect to see the dependencies of the reconnection rate on viscosity until the Prandtl number is less than unity since the cascade can proceed to scales smaller than the current sheet thickness affecting the rate of local reconnection. This is the case we are studying here. For large Prandtl numbers the reconnection may be suppressed as magnetic field becomes more laminar at sufficiently large scales. However, two main factors of the LV99 model contributing to the enhancement of the reconnection rate in the presence of turbulence are that the turbulence allows for multiple reconnection events acting simultaneously and secondly, the turbulence should broaden the outflow region to the widths corresponding to the scales much larger than the dissipation scale and comparable to the injection scale allowing to remove more reconnected flux. While the first factor could be affected by the large viscosity, the second is still valid if the injection scale is much larger than the dissipation scale. The case when the effective Prandtl numbers are larger than unity due to the presence of neutrals is discussed in Lazarian et al. (2004). We plan to study the dependence on the Prandtl number in our next publication.

The LV99 model predicts that the reconnection speed should grow with the injection scale as $V_{r e c} \sim l_{i n j}$. However, some of our numerical tests show a weaker dependence, $V_{\text {rec }} \sim l_{i n j}^{3 / 4}$. The origin of this discrepancy is unclear. Our model of how weak isotropic turbulence evolves cascades into strong turbulence may be too naive. In particular, the approximation that the parallel wavelength is unchanged during this process may be simplistic. In addition, we may simply not have sufficient dynamic range between the dimensions of the subvolume where turbulence is excited and the thickness of the Sweet-Parker layer. Corrections at either end could result in the appearance of a shallower dependence on the driving scale. The true nature of this discrepancy should be explored in future work.

Even though these numerical simulations allow us to study reconnection in the presence of turbulence for a limited range of magnetic Reynolds numbers (in this pa- per $R_{m}<10^{3}$ ), the results provide good testing of the relations derived by LV99. The strong dependence of $V_{\text {rec }}$ on the injection scale, when scaled to the real conditions of the interstellar medium, shows a dramatic enhancement of the reconnection speed, even in the situation of an almost perfectly frozen magnetic field in the medium. This allows for fast reconnection with the characteristic time comparable to one Alfvén time unit.

\subsection{Implications of the LV99 Model}

Reconnection is one of the most fundamental processes involving magnetic fields in conducting fluids or plasmas. Therefore, the identification of a robust process responsible for reconnection has many astrophysically important consequences. Below we shall mention a few selected implications of the successful validation of the LV99 model.

Following Zweibel \& Yamada (2009) we note that solar flares inspired much of the earlier research on reconnection (see Pneuman 1981; Bastian et al. 1998; Hudson 2008). As the plasma involved is substantially rarefied, the restrictive conditions for the collisionless reconnection are satisfied in this particular environment. Cassak et al. (2005) stated that bistable Hall reconnection can be important in this case. Stochastic reconnection, as we discuss below, provides an alternative explanation.

Indeed, an important prediction of the LV99 model is related to the reconnection instability that arises in the situation when the initial structure of the flux prior to reconnection is laminar. Reconnection at the Sweet-Parker rate is negligible. This allows magnetic flux to accumulate. However, when the degree of stochasticity exceeds a threshold value, the reconnection itself should excite more turbulence, creating a positive feedback resulting in a flare (see Lazarian \& Vishniac 2008). The instability is a generic property of laminar field reconnection in both collisionless and collisional environments. Referring to the Sun, one may speculate that the difference between gradual and eruptive flares arises from the original state of magnetic field prior to the flare. In the case when the magnetic field is sufficiently turbulent the accumulation of magnetic flux does not happen and the flare is gradual. Similarly, the observed spatial spread of energy release during solar flares may be due to the spread of the region of turbulent fields once reconnection is initiated at one place. Further research is necessary for testing these ideas.

In the Sweet-Parker model reconnection can accelerate charged particles, e.g. due to the electric field in the reconnection region (see Litvinenko 2003). However, the speed of Sweet-Parker reconnection is negligible for most astrophysical environments, thus the transfer of energy from the magnetic field to particles is absolutely negligible, if reconnection follows the Sweet-Parker predictions.

It is interesting to notice that the first-order Fermi acceleration process is intrinsic to the LV99 model of reconnection. Consider a particle entrained on a reconnected magnetic field line (see Fig1). This particle may bounce back and forth between magnetic mirrors formed by oppositely directed magnetic fluxes moving towards each other with the velocity $V_{R}$. Each bounce will increase the energy of a particle in a way consistent with the requirements of the first-order Fermi 
process $^{11}$ (de Gouveia dal Pino \& Lazarian 2001, 2003, 2005; Lazarian 2006). This is in contrast to the secondorder Fermi acceleration that is frequently discussed in terms of accelerating particles by turbulence generated by reconnection (La Rosa et al. 2006).

An interesting property of this acceleration mechanism is that it is potentially testable observationally, since the resulting spectrum of accelerated particles is different from that arising from a shock. de Gouveia dal Pino \& Lazarian (2001, 2005) used this mechanism of particle acceleration to explain the synchrotron power-law spectrum arising from the flares of the microquasar GRS 1915+105. The mechanism is similar to the acceleration mechanism that was discussed later by Drake et al. (2006b). Drake et al. (2006b) considered the acceleration of electrons and, similarly, to the Matthaeus et al. (1984), assumed that the acceleration happens within $2 \mathrm{D}$ contracting loops.

To enable sustainable dynamo action and, for example, generate a galactic magnetic field, it is necessary to reconnect and rearrange magnetic flux on a scale similar to a galactic disc thickness within roughly a galactic eddy turnover time $\left(\sim 10^{8}\right.$ years $)$. This implies that reconnection must occur at a substantial fraction of the Alfvén velocity. The preceding arguments indicate that such reconnection velocities should be attainable if we allow for a realistic magnetic field structure, one that includes both random and regular fields (see Hanasz et al. 2004, e.g.). This does solve one part of the problem of dynamo. The other part is related to magnetic helicity conservation (see Vishniac et al. 2003).

Interestingly enough, while earlier on we criticized the concept of turbulent diffusivity as ill founded, we should mention that the robust reconnection of turbulent fields can allow parcels of magnetized fluid to move being less constrained by the surrounding magnetic fields. This allows matter and heat to transfer with the rates determined by a simple turbulent diffusion coefficient $\sim v_{\text {turb }} l_{\text {turb }}$ which for typical parameters of plasma in galaxy clusters exceed the rates of thermal diffusion of electrons Lazarian (2006). An important distinction remains however. Reconnection happens in thin current sheets, involving a negligible fraction of the magnetic field. Unlike a large effective resistivity, fast reconnection cannot violate magnetic helicity conservation.

Finally, LV99 showed that fast reconnection of stochastic magnetic field makes the models of strong MHD turbulence self-consistent. Indeed, critical balance in the GS95 model requires the existence of eddy-type motions perpendicular to the magnetic field. In the absence of reconnection this would result in unresolved knots that should drain energy from the cascade. The estimates in LV99 showed that the rates of reconnection predicted by the model are sufficient to resolve magnetic knots within one period.

\subsection{Final Remarks}

\footnotetext{
11 Another way of understanding the acceleration of energetic particles in the reconnection process above is to take into account that the length of magnetic field lines is decreasing during reconnection. As a result, the physical volume of the energetic particles entrained on the field lines is shrinking. Thus, due to Louiville theorem, their momentum should increase to preserve the constancy of the phase volume.
}

In this paper we have considered reconnection for weak MHD turbulence. What would happen if the turbulence is strong, i.e. when the injection of turbulent energy happens with superAlfvénic velocities? In this situation we expect the turbulent cascade to proceed initially in a way similar to ordinary hydrodynamics with strong hydrodynamic motions easily bending magnetic field lines. However, at some small scale we expect the magnetic field to be dynamically important and resist bending. Our reconnection results should be applicable to turbulence starting at this scale.

The successful numerical testing of the turbulent reconnection model presented in this paper appeals for more studies in this direction. It is important to stress that, unlike brute force simulations, we were able to test the LV99 model of three dimensional reconnection giving support to the idea that we could reliably extend our results to much higher Lundquist numbers. The problems for future studies cover the importance of compressibility in this type of reconnection, since a substantialfraction of observed interstellar medium is supersonic. Another interesting question is the ability of turbulent reconnection to self-sustaining or to generate turbulence by itself ${ }^{12}$. These are problems we hope to address in the future.

\section{SUMMARY}

In this article we put to the test the LV99 model of reconnection by investigating the influence of weak turbulence on the reconnection process using 3D numerical simulations. The turbulence is weak in the sense that the injection velocities are less than the Alfvén speed and the magnetic field direction is only weakly perturbed by turbulence. It is strong in the sense of producing a strongly nonlinear cascade of energy. In our study we experimented with different boundary conditions and with different ways of measuring the reconnection speed. We analyzed the dependence of the reconnection speed on the turbulence injection power, on the injection scale, as well as on Ohmic and anomalous resistivity. We found that:

- Turbulence in 3D drastically changes the topology of the magnetic field near the interface of oppositely directed magnetic field lines. These changes include the fragmentation of the current sheet, favoring multiple simultaneous reconnection events, as well as a substantial increase in the thickness of the outflow of reconnected magnetic flux and matter.

- The intuitive measure of reconnection defined as the inflow of magnetic field velocity corresponds well, in the condition of stationary reconnection, to a more rigorously defined reconnection measure that we introduced. The two measures show the same dependencies of $V_{r e c}$ on the power $P_{i n j}$ and injection scale $l_{i n j}$ of the turbulence, regarding the different scheme used to solve MHD equations and different sets of initial parameters.

12 While, as we argued earlier, turbulence is a natural state of most of the astrophysical fluids, it is important to know whether the reconnection is determined by the preexisting level of turbulence, or it can get self-accelerated. 
- The reconnection rate is determined by the thickness of the outflow region. For large scale turbulence, the reconnection rate depends on the amplitude of fluctuations and injection scale as $V_{\text {rec }} \sim$ $P_{i n j}^{1 / 2} \sim V_{l}^{2}$ which corresponds to LV99 predictions.

- The reconnection rate grows with the turbulence injection scale, which qualitatively corresponds to the LV99 predictions. However, in some cases the rate of growth is better approximated by $V_{\text {rec }} \sim$ $l_{\text {inj }}^{3 / 4}$ scaling rather than $V_{\text {rec }} \sim l_{\text {inj }}$ predicted in LV99. The difference may stem from details of the initial weak cascade of energy, or from limitations in the dynamic range available for study.

- Reconnection in the presence of weak turbulence is not sensitive to Ohmic resistivity, which corresponds to the LV99 prediction that the reconnection of weakly stochastic magnetic fields are generically fast.

- The introduction of anomalous resistivity does not change the rate of reconnection of a weakly stochastic field either, which supports the assertion in LV99 that in the presence of magnetic field stochasticity, which is generic for most of astrophysical environments, plasma effects, e.g. collisionless effects, are irrelevant in determining reconnection speeds.

- The strength of the guide field does not change reconnection rates for similar rates of turbulent energy injection. Thus fast reconnection is possible for generic configurations when magnetic bundles intersect each other at arbitrary angles.

The research of GK and AL is supported by the Center for Magnetic Self-Organization in Laboratory and Astrophysical Plasmas and NSF Grants ATM-06-48699 and AST-08-08118. The work of ETV is supported by the National Science and Engineering Research Council of Canada. Part of this work was made possible by the facilities of the Shared Hierarchical Academic Research Computing Network (SHARCNET:www.sharcnet.ca). This research also was supported by the National Science Foundation project TG-AST090078 through TeraGrid resources provided by Texas Advanced Computing Center (TACC:www.tacc.utexas.edu). We would like to thank Mel Goldstein, Paul Cassak, Bill Matthaeus, Dmitri Uzdenski, Ellen Zweibel and Masaaki Yamada for fruitful discussions. Helpful suggestions of the anonymous referee are acknowledged.

\section{REFERENCES}

Alvelius, K. 1999, Physics of Fluids, 11, 1880

Armstrong, J. W., Rickett, B. J., \& Spangler, S. R. 1995, ApJ, 443, 209

Bastian, T. S., Benz, A. O., \& Gary, D. E. 1998, ARA\&A, 36, 131

Beck, R. 2002, in ASP Conf. Ser. 275, Disks of Galaxies: Kinematics, Dynamics and Perturbations, ed. E. Athanassoula, A. Bosma, \& R. Mujica (San Francisco, CA:ASP), 331

Beresnyak, A., \& Lazarian, A. 2006, ApJ, 640, L175

Beresnyak, A., \& Lazarian, A. 2008a, ApJ, 682, 1070

Beresnyak, A., \& Lazarian, A. 2008b, arXiv:0812.0812

Beresnyak, A., \& Lazarian, A. 2009, ApJ, submitted

Bhattacharjee, A., \& Hameiri, E. 1986, Physical Review Letters, 57, 206

Bhattacharjee, A., Ma, Z. W., \& Wang, X. 2003, in Turbulence and Magnetic Fields in Astrophysics, Lecture Notes in Physics, 614, 351

Bhattacharjee, A. 2004, ARA\&A, 42, 365

Biskamp, D. 1996, Ap\&SS, 242, 165

Biskamp, D. 2000, Magnetic Reconnection in Plasmas (Cambridge, UK:Cambridge University Press)

Biskamp, D. 2003, Magnetohydrodynamic Turbulence (Cambridge, UK:Cambridge University Press)

Blackman, E. G., \& Field, G. B. 2008, MNRAS, 386, 1481

Boldyrev, S. 2005, ApJ, 626, L37

Boldyrev, S. 2006, Phys. Rev. Lett., 96, 11, 5002

Cassak, P. A., Shay, M. A., \& Drake, J. F. 2005, Phys. Rev. Lett. 95, 23, 5002

Chandran, B. D. G. 2008, ApJ, 685, 646

Cho, J., \& Vishniac, E. 2000, ApJ, 539, 273

Cho, J., Lazarian, A., \& Vishniac, E. T. 2002, ApJ, 564, 291

Cho, J., \& Lazarian, A. 2002, Phys. Rev. Lett., 88, 24, 5001

Cho, J., \& Lazarian, A. 2003, MNRAS, 345, 325

Ciaravella, A., \& Raymond, J. C. 2008, ApJ, 686, 1372

Courant, R., Friedrichs, K., \& Lewy, H., 1928, Mathematische Annalen, 100, 32

Crutcher, R. M. 1999, ApJ, 520, 706

Daughton, W., Scudder, J., \& Karimabadi, H. 2006, Physics of Plasmas, 13, 2101

Dahlburg, R. B., Antiochos, S. K., \& Zang, T. A. 1992, Physics of Fluids B, 4, 3902

Dahburg, R. B. \& Karpen, T. 1994, ApJ, 434, 766

Dahlburg, R. B. 1997, Journal of Plasma Physics, 57, 35
Del Zanna, L., Bucciantini, N., \& Londrillo, P. 2003, A\&A, 400, 397

Diamond, P. H., \& Malkov, M. 2003, Physics of Plasmas, 10, 2322 Drake, J. F., Swisdak, M., Che, H., \& Shay, M. A. 2006a, Nature, 443,553

Drake, J. F., Swisdak, M., Schoeffler, K. M., Rogers, B. N., \& Kobayashi, S. 2006b, Geophys. Res. Lett., 33, 13105

Elmegreen, B. G., \& Scalo, J. 2004, ARA\&A, 42, 211

Eyink, G. L., \& Aluie, H. 2006, Physica D Nonlinear Phenomena, 223,82

Ferraro, N. M., \& Rogers, B. N. 2004, Physics of Plasmas, 11, 4382 Fitzpatrick, R. 2004, Physics of Plasmas, 11, 937

Galama, T. J., et al. 1998, Nature, 395, 670

Goldreich, P., \& Sridhar, S. 1995, ApJ438, 763, (GS95)

Gogoberidze, G. 2007, Physics of Plasmas, 14, 2, 2304

de Gouveia dal Pino, E., \& Lazarian, A. 2001, ApJ, 560, 358

de Gouveia dal Pino, E., \& Lazarian, A. 2003, arXiv:astro-ph/0307054

de Gouveia dal Pino, E., \& Lazarian, A. 2005, A\&A, 441, 845

Hameiri, E., \& Bhattacharjee, A. 1987, Physics of Fluids, 30, 1743

Hanasz, M., Kowal, G., Otmianowska-Mazur, K., \& Lesch, H. 2004, ApJ, 605, L33

Harten, A., Lax, P. D., \& van Leer, B. 1983, SIAM Rev. 25, 35

Hedstrom, G. W. 1979, Journal of Computational Physics, 30, 222

Heitsch, F., \& Zweibel, E. G. 2003a, ApJ, 583, 229

Heitsch, F., \& Zweibel, E. G. 2003b, ApJ, 590, 291

Higdon, J. C. 1984, ApJ, 285, 109

Hudson, H. S. 2008, Heliophysics II. Energy Conversion Processes, ed. C. J. Schrijver \& G. L. Siscoe

Iroshnikov, P. S. 1963, AZh, 40, 742

Jacobson, A. R., \& Moses, R. W. 1984, Phys. Rev. A, 29, 3335

Kim, E.-J., \& Diamond, P. H. 2001, ApJ, 556, 1052

Kowal, G., Lazarian, A., \& Beresnyak, A. 2007, ApJ, 658, 423

Kowal, G., \& Lazarian, A. 2007, ApJ, 666, L69

Kraichnan, R. H. 1965, Physics of Fluids, 8, 1385

Kulpa-Dybel, K., Otmianowska-Mazur, K., Kowal, G., Lazarian, A., Vishniac, E. T., 2009, in prep.

La Rosa, T. N., Shore, S. N., Joseph, T., Lazio, W., \& Kassim, N. E. 2006, Journal of Physics Conference Series, 54, 10

Lazarian, A., \& Vishniac, E. T. 1999, ApJ, 517, 700, (LV99)

Lazarian, A., Vishniac, E. T., \& Cho, J. 2004, ApJ, 603, 180, (LVC04) 
Lazarian, A. 2006, Astronomische Nachrichten, 327, 609

Lazarian, A., \& Vishniac, E. T. 2008, in Revista Mexicana de Astronomía y Astrofísica Conf. Ser., in press, arXiv:0812.2019

Levy, D., Puppo, G., \& Russo, G. 1999, Mathematical Modelling and Numerical Analysis, 33, 547

Lithwick, Y., \& Goldreich, P. 2001, ApJ, 562, 279

Lithwick, Y., Goldreich, P., \& Sridhar, S. 2007, ApJ, 655, 269

Litvinenko, Y. E. 2003, Sol. Phys., 216, 189

Liu, X.-D., \& Osher, S. 1998, Journal of Computational Physics, 141,1

Londrillo, P., \& Del Zanna, L. 2000, ApJ, 530, 508

Maron, J., \& Goldreich, P. 2001, ApJ, 554, 1175

Masuda, S., Kosugi, T., Hara, H., Tsuneta, S., \& Ogawara, Y. 1994, Nature, 371, 495

Matthaeus, W. H., Ambrosiano, J. J., \& Goldstein, M. L. 1984, Phys. Rev. Lett., 53, 1449

Matthaeus, W. H., \& Lamkin, S. L. 1985, Physics of Fluids, 28, 303

Matthaeus, W. H., \& Lamkin, S. L. 1986, Physics of Fluids, 29, 2513

McKee, C. F., \& Ostriker, E. C. 2007, ARA\&A, 45, 565

Mignone, A. 2007, Journal of Computational Physics, 225, 1427

Moffat, H. K. 1978, Magnetic Field Generation in Electrically conducting Fluids, (London, UK/New York, NY:Cambridge University Press)

Montgomery, D., \& Turner, L. 1981, Physics of Fluids, 24, 825

Ostriker, E. C., Stone, J. M., \& Gammie, C. F., 2001, ApJ, 546, 980

Parker, E. N. 1957, J. Geophys. Res., 62, 509

Petschek, H. E. 1964, in Conf. Proc. of the AAS-NASA Symposium, Physics of Solar Flares, ed. W. H. Hess, (Washington, DC:NASA Science and Technical Information Division), 425

Pneuman, G. W. 1981, in Solar Flare Magnetohydrodynamics, ed. E. R. Priest, (New York, NY:Gordon and Breach Science Publishers), 379

Priest, E., \& Forbes, T. 2000, Magnetic Reconnection, (Cambridge, UK:Cambridge University Press)
Schlickeiser, R. 2004, Physics of Plasmas, 11, 5532

Shay, M. A., Drake, J. F., Denton, R. E., \& Biskamp, D. 1998, J. Geophys. Res., 103, 9165

Shay, M. A., Drake, J. F., \& Swisdak, M. M. 2004, Physics of Plasmas, 11, 2199

Shebalin, J. V., Matthaeus, W. H., \& Montgomery, D. 1983, Journal of Plasma Physics, 29, 525

Shibata, K., \& Tanuma, S. 2001, Earth, Planets, and Space, 53, 473

Shu, F. H., Galli, D., Lizano, S., \& Cai, M. 2006, ApJ, 647, 382

Shu, F. H., Galli, D., Lizano, S., Glassgold, A. E., \& Diamond, P. H. 2007, ApJ, 665, 525

Smith, D., Ghosh, S., Dmitruk, P., \& Matthaeus, W. H. 2004, Geophys. Res. Lett., 31, L02805

Speiser, T. W. 1970, Planet. Space Sci., 18, 613

Strauss, H. R. 1986, Physics of Fluids, 29, 3668

Strauss, H. R. 1988, ApJ, 326, 412

Sturrock, P. A. 1966, Nature, 211, 695

Sweet, P. A. 1958, Conf. Proc. IAU Symposium 6, Electromagnetic Phenomena in Cosmical Physics, ed. B. Lehnert, (Cambridge, UK:Cambridge University Press), 123

Tassis, K., \& Mouschovias, T. C. 2005a, ApJ, 618, 769

Tassis, K., \& Mouschovias, T. C. 2005b, ApJ, 618, 783

Vishniac, E. T., \& Lazarian, A. 1999, ApJ, 511, 193, (VL99)

Vishniac, E. T., Lazarian, A., \& Cho, J. 2003, Turbulence and Magnetic Fields in Astrophysics, Lecture Notes in Physics, 614, 376

Watson, P. G., Oughton, S., \& Craig, I. J. D. 2007, Physics of Plasmas, 14, 032301

Waelbroeck, F. L. 1989, Physics of Fluids B, 1, 2372

Wang, X., Bhattacharjee, A., \& Ma, Z. 2001, Phys. Rev. Lett., 87, 265003

Yamada, M. 2007, Physics of Plasmas, 14, 5, 8102

Zweibel, E., \& Yamada, M. 2009, ARA\&A, in press 